\title{
Influence of attachment pressure and kinematic configuration on pHRI with wearable robots
}

\author{
André Schiele*a and Frans C.T. van der Helm ${ }^{\mathrm{b}}$ \\ ${ }^{a}$ Mechanical Engineering Department, Automation and Robotics Section, European Space Agency, ESA/ESTEC, \\ Noordwijk, The Netherlands, ${ }^{b}$ Faculty of Mechanical Engineering, BioMechanical Engineering Department, \\ Delft University of Technology, Delft, The Netherlands
}

(Received 12 December 2008; final version received 10 March 2009)

\begin{abstract}
The goal of this paper is to show the influence of exoskeleton attachment, such as the pressure on the fixation cuffs and alignment of the robot joint to the human joint, on subjective and objective performance metrics (i.e. comfort, mental load, interface forces, tracking error and available workspace) during a typical physical human-robot interaction (pHRI) experiment. A mathematical model of a single degree of freedom interaction between humans and a wearable robot is presented and used to explain the causes and characteristics of interface forces between the two. The pHRI model parameters (real joint offsets, attachment stiffness) are estimated from experimental interface force measurements acquired during tests with 14 subjects. Insights gained by the model allow optimisation of the exoskeleton kinematics. This paper shows that offsets of more than $\pm 10 \mathrm{~cm}$ exist between human and robot axes of rotation, even if a well-designed exoskeleton is aligned properly before motion. Such offsets can create interface loads of up to $200 \mathrm{~N}$ and $1.5 \mathrm{Nm}$ in the absence of actuation. The optimal attachment pressure is determined to be $20 \mathrm{mmHg}$ and the attachment stiffness is about $300 \mathrm{~N} / \mathrm{m}$. Inclusion of passive compensation joints in the exoskeleton is shown to lower the interaction forces significantly, which enables a more ergonomic pHRI.
\end{abstract}

Keywords: exoskeleton; ergonomics; physical human-robot interaction; interaction force; subjective and objective measures

\section{Introduction}

Exoskeletons are subject to intense interest and research at the current time, for a large field of applications that spans from haptics and fundamental haptic device research (Frisoli et al. 2005) over bilateral tele-robotics (Bergamasco et al. 1994; Schiele et al. 2006) and defence applications (Kazerooni and Steger 2006; Zoss and Kazerooni 2006) up to the field of robotic physical therapy (Tsagarakis and Caldwell 2003; Riener et al. 2005; Carignan et al. 2007; $\mathrm{Nef}$ et al. 2007). All types of wearable robots must be safe, comfortable and be able to smoothly interact with the human user. Safe physical human-robot interaction (pHRI) is difficult to achieve and to quantify, and therefore is still a relatively new and important area of research in the field of robotics (Alami et al. 2006).

Two of the most important aspects influencing comfort and safety in wearable robots are the actuation and control and the kinematic design of the movable structure.

So far, many researchers have published novel actuation concepts such as compliant drives (Pratt and Williamson 1995; Zinn et al. 2004) or antagonist actuators, as well as advanced control architectures (Raibert and Craig 1981; Hogan 1985) that help to substantially improve the safety of human-machine interaction for wearable robots. Hybrid position and force controllers are now being developed for exoskeletons used in physical therapy that make the robots safer than earlier purely position-controlled devices.

An area to which too little attention has been paid, however, is the mechanical and kinematic design of wearable robots for optimal pHRI. Currently, each designer of a wearable system optimises his system according to his personal understanding and experience rather than consulting a vast background of existing knowledge about this field. According to the knowledge of the authors, no study so far has extensively addressed the topic of ergonomics and pHRI for wearable robots, let alone provided a theoretical framework for detailed analysis. We have addressed this field extensively as part of a larger investigation (Schiele 2008d).

This is despite the fact that kinematic mismatch between a human and a robot can cause injury or at least significant discomfort for the operators, as was reported in Colombo et al. (2000a, 2000b). For robotic physical therapy, the field in which pHRI is certainly required to be the most natural and least constraining, kinematic mismatches between the axes of motion of the robot and the human were shown to reduce the effect of therapy by altering the natural patterns of movement (Hidler and Wall 2005). Neckel et al. (2007) report that natural joint moments are altered in stroke patients if they train with a robotic orthosis that

*Corresponding author. Email: andre.schiele@esa.int 
is not well harmonised with human physiology. Causes of such kinematic disparity can be joint centre of rotation offsets between human and robot limbs and oversimplified kinematic structures of the wearable robot. The LOKOMAT orthesis, for instance, features a somewhat non-ergonomic pHRI due to the absence of degrees of freedom for pelvic motion and the difficulty of aligning the principal axes of motion correctly. All types of kinematic offsets between human and robot create disturbance forces (sometimes also called interface or interaction forces) at the human-robot interface.

Also, in other wearable robot applications, improved kinematic design and, in particular, design for the human will be important. Presence of disturbance forces in haptic devices, for instance, would be detrimental to their performance if the created interaction forces are in the same order of magnitude as the feedback forces. A mechanically more 'transparent' device design alone (transparent in the sense that no disturbing forces are created during movement) could improve the perception of sensory feedback and, thus, device performance.

It is crucial to note that the causes and characteristics of force artefacts in pHRI stemming from kinematic inequality and from non-ideal attachment between human and robot are poorly explained and covered in literature today. Effects of interaction forces on subjectively perceived metrics are not known, nor are the objective effects explained on mathematical grounds. This paper aims to link subjective and objective performance metrics acquired during an experiment with a wearable robot to the geometry of the combined human-exoskeleton system. A theoretical pHRI model for one degree of freedom (d.o.f.) interaction is proposed that allows interpreting the measured data and explaining cause and characteristics of interface force creation. A solution to the problem of kinematic alignment is presented, which is based on inclusion of passive compensatory joints in the wearable robot structure. The experiment presented is conducted with and without such compensatory joints to analyse their effect on pHRI.

\section{Rationale}

Recently we have presented a novel design paradigm for human-centred exoskeleton kinematics (Schiele and van der Helm 2006). We have hypothesised there that smooth pHRI is disturbed by creation of interaction loads during movement which stem from offsets between the main axes of rotation of robot and human limbs. In particular, we have shown that macro-misalignments, resulting from multiple d.o.f. mismatch between human and robot, e.g. between a 3-d.o.f. shoulder interface of a wearable robot and a real human shoulder girdle (with more than 5 d.o.f.), can be compensated effectively by a wearable robot structure according to the kinematics paradigm presented in Schiele and van der Helm (2006). We have hypothesised that inclusion of passive compensatory joints for a single d.o.f. interaction with human limbs can compensate interaction forces experienced by the user stemming from micro-misalignments, which are offsets of the main rotary joints of human and robot. Figure 1(a) illustrates the concept of interaction force $F_{\mathrm{d}}$ creation during movement, based on offsets between the human limb centre of rotation $\mathrm{ICR}_{\mathrm{h}}$ and the wearable robot limb's centre of rotation $\mathrm{CR}_{\mathrm{e}}$. In Figure 1(b), the concept of passive compensatory joints is illustrated. It is not known, however, what the magnitude of such micro-misalignments is in a typical task and how exactly the misalignments influence the creation of constraint forces. We have therefore developed an analytical model of interaction force depending on the geometric attachment parameters between the exoskeleton and the limb. This model will be used in (a)

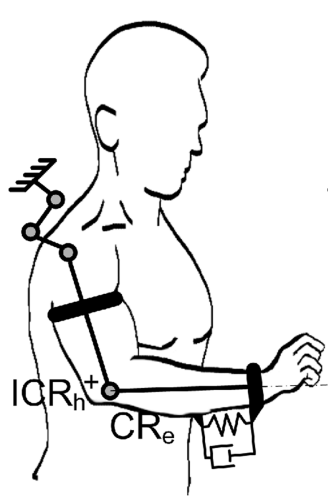

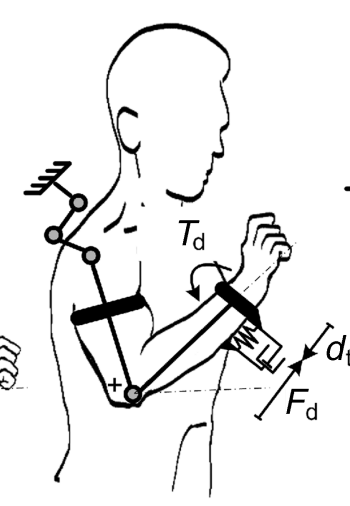

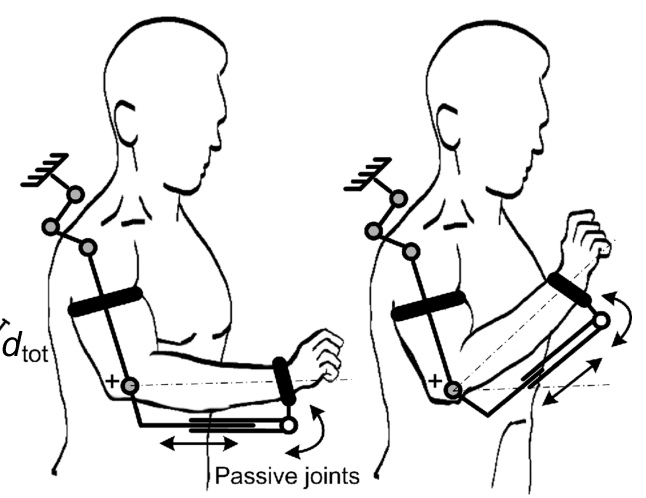

Figure 1. Offsets between the instantaneous centre of rotation of a human arm $\mathrm{ICR}_{\mathrm{h}}$ and the centre of rotation of an exoskeleton $\mathrm{CR}_{\mathrm{e}}$ can create constraint displacements $d_{\text {tot }}$ that result in perceived interaction forces $F_{\mathrm{d}}$ and torques $T_{\text {drot }}$ during movement (a). In (b), an exoskeleton kinematic structure is shown that includes passive compensation joints aiming to reduce such interface forces. 


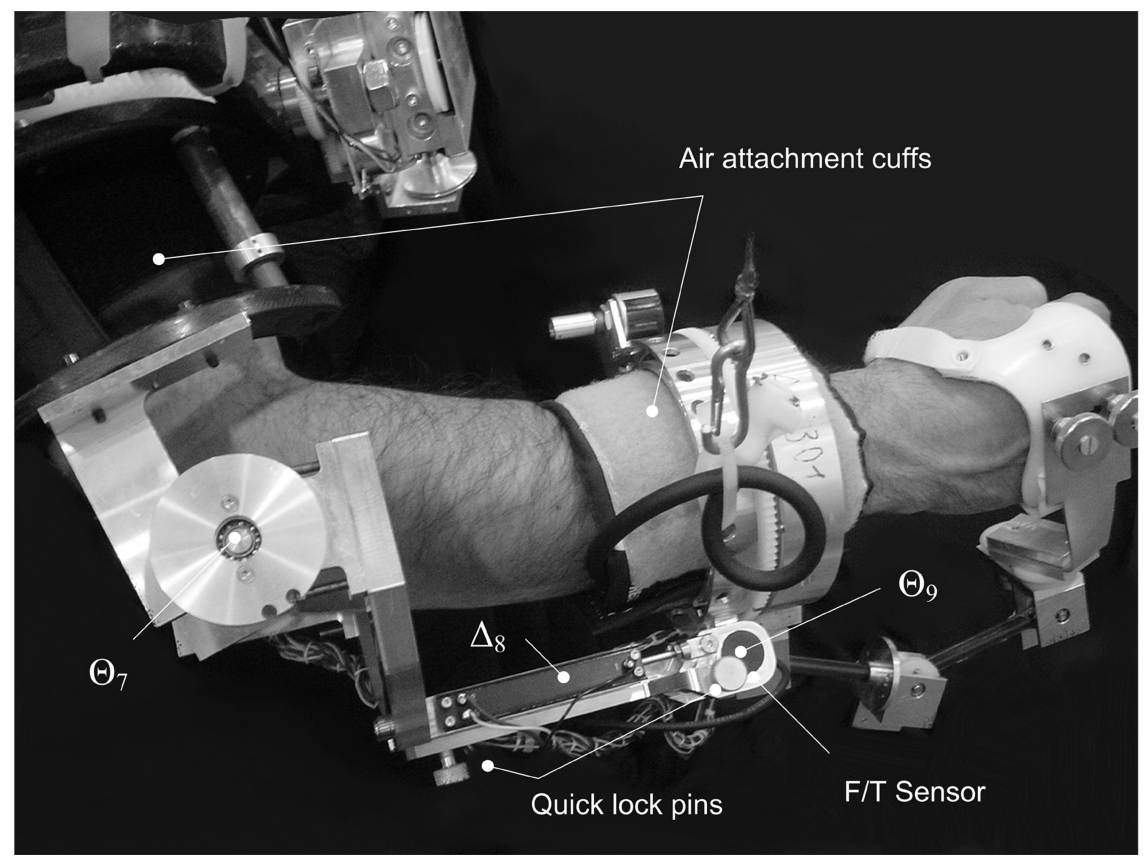

Figure 2. Overview of the elbow articulation of the ergonomic EXARM exoskeleton used to conduct the experiments. $\Theta_{7}$ is the main elbow rotation axis of the exoskeleton. The effect of its alignment on the creation of interaction forces was a main measure in the experiment. $\Delta_{8}$ is a linear and $\Theta_{9}$ a rotary compensation joint. The compensation joints can be locked by quick lock pins to emulate a non-ergonomic device design.

this paper for parameter estimation of the pHRI of our subjects.

It is the goal of this paper to quantify the effect of joint misalignments and to evaluate its influence on creation of interface loads. In particular, it is the goal to relate subjective perception and objective performance metrics to (1) the attachment pressure between the exoskeleton and the human limb and to (2) the presence/absence of kinematic compensatory joints in the mechanical structure of the exoskeleton. It shall be investigated whether the geometry of the attachment between human and robot influences the creation of interaction forces and how they can be optimally reduced by incorporating compensatory joints into a wearable exoskeleton.

\section{Method}

An experiment was conducted in which a group of subjects was asked to perform a proportional visual-tracking task with their elbow while wearing the ESA EXARM exoskeleton (Schiele and van der Helm 2006; Schiele 2008c). The kinematic structure of the exoskeleton as well as the interface pressure on the operator were altered between experiment runs.

\subsection{Experimental setup}

The experiment was carried out with the elbow interface of the EXARM exoskeleton. The exoskeleton was attached to the operator's upper arms and forearms by means of two inflatable air cushions and gravity-balanced by a cable system with compensation masses (Figure 2). The EXARM comprises three joints for the elbow articulation. The first joint, $\Theta_{7}$, is the main joint for flexion-extension of the elbow (aligned with the anatomical elbow flexion-extension axis), the second, $\Delta_{8}$, is a linear passive compensation joint and the third, $\Theta_{9}$, a rotary passive compensation joint. [The joint naming corresponds to the previous description in Schiele and van der Helm (2006).]

The exoskeleton's kinematic structure is similar to the one illustrated in Figure 1(b). In Schiele and van der Helm (2006), we have shown that the exoskeleton is well designed in its kinematic parameters. For this experiment, joints $\Delta_{8}$ and $\Theta_{9}$ were re-designed. Quick lock pins were incorporated that allow locking of the passive joints to emulate the kinematic structure of wearable robots without compensation joints. If the joints are locked, the exoskeleton is similar to the one illustrated schematically in Figure 1(a). Still, it can be adjusted prior to experiments to properly fit the wearer.

\subsection{Experiment protocol}

The experiment was conducted with seven male and seven female candidates (stature: $1.75 \mathrm{~m} \pm 0.09 \mathrm{~m}$; mass: 68.7 $\mathrm{kg} \pm 12.8 \mathrm{~kg}$ ) that were untrained and not informed about the detailed scope of this experiment. Each subject was asked during 12 experiment trials to visually track a random 
crested multisine (multisinusoiudal) signal, the target signal $v$, on a computer screen with the motion of their elbow. The angle $\beta$ on the EXARM elbow joint $\left(\Theta_{7}\right)$ was measured and displayed along with $v$ as moving bars on a computer screen. The instantaneous tracking error was shown as a third bar on the screen, to give some feedback of the current tracking performance. The duration of each trial was $60 \mathrm{~s}$. The target signal demanded elbow flexion from $0^{\circ}$ to $90^{\circ}$ with frequencies ranging between 0.05 to $0.35 \mathrm{~Hz}$. Between the 12 trials, the kinematic setting of the exoskeleton as well as the interface pressure between the fixation cuffs of the exoskeleton and the human limb were varied randomly by the experimenter. Each combination of factors was tested once per subject and the subjects were blinded to the experiment conditions. The kinematic settings were called 'locked' when all passive compensatory joints of the exoskeleton were fixed, and 'unlocked' when all passive compensatory joints were free to move. The interface pressure $P$ was varied between 10 and $60 \mathrm{mmHg}$ in steps of 10 $\mathrm{mmHg}$, always equal for the two cuffs. During the trials, the multisine target signal $\nu$, the displacements $\beta$ (of joint $\Theta_{7}$ ), $d_{\text {lin }}$ (of joint $\Delta_{8}$ ) and $\Theta_{\text {rot }}$ (of joint $\Theta_{9}$ ), the interaction force $F_{\mathrm{d}}$ and the moment $T_{\mathrm{d}}$ (Figure 1) were recorded at $1 \mathrm{kHz}$ sampling frequency. While the joint motion was measured with high precision potentiometers, the interaction loads were measured with a 6-d.o.f. force sensor (ATI Nano Series) inserted between the forearm cuff of the exoskeleton and the exoskeleton structure (Figure 2). All signals were anti-aliased by analogue filters to limit noise to below the quantisation noise of the $\mathrm{A} / \mathrm{D}$ converters (12 bit).

After each trial, the subjects were asked to rate a subjective questionnaire on a visual rating scale with a pen. They marked their rating on eight linear rating scales that ranged from 0 (low) to 100 (high) points for (1) 'comfort' (definition: How comfortable was this setting of the exoskeleton during movement?), (2) 'motion hindrance' (definition: How much hindrance to the movement did you experience?) and (3-8) the six NASA TLX rating scales of physical demand (PD), mental demand (MD), temporal demand (TD), operator performance (OP), effort (EF) and frustration level (FR). The definitions presented to the subjects were adopted from Hart and Staveland (1988). After all 12 trials, the weighting factors for the TLX rating scales were acquired for each subject by pair-wise comparisons and computed into an overall set of group weights. Next, the most comfortable attachment pressures were determined per subject by inflating the cuffs and asking the most comfortable setting. The most comfortable pressures were then noted by the experimenter.

To get the subjects used to the set-up before the real experiment, a five-trial training session had been carried out. Pilot experiments had confirmed that this was sufficient to get the subjects used to the task. The test runs were conducted with a different input signal and no variation of the attachment and kinematic properties. This accustomed the subjects to the task and to the rating on the subjective rating scales.

After each experiment trial, the exoskeleton was reattached such that the exoskeleton elbow flexion joint $\left(\Theta_{7}\right)$ aligned well with the human elbow flexion axis. The fixation position was determined visually and then fine-tuned by some movement of the exoskeleton articulation on the arm before inflating the air cushions to fix the device.

All tracking movements were conducted with a horizontally elevated arm in a horizontal plane in order to remove the effect of gravity on the force measurements as far as possible. This way, the measured axes did not show influence on gravitational force.

\subsection{Statistical design and analysis}

A series of statistical tests was performed to determine the effects of the experiment conditions (independent variables) on the output measures (dependent variables). All statistical tests were factored analyses of variance (ANOVA) that test for equality of sample population means.

\subsubsection{Independent variables}

The experiment features two main independent factors, which are (F1) the kinematic condition, with two levels 'locked' and 'unlocked' (hereafter called $L$ and $U$, respectively), and (F2), the interface pressure with six levels ranging from 10 to $60 \mathrm{mmHg}$. The subjects were used as a third factor (F3) in some presented statistical analyses, in order to take into account the variability between the subjects that may provide a large part of the overall measured variance.

\subsubsection{Dependent variables}

Objective as well as subjective performance measures are analysed in this study. Both groups consist of mean output measures that are averaged over each full experiment run (1 min at $1 \mathrm{kHz}$ sampling). For the objective metrics, those measures are (1) the mean absolute interaction force $\left|\bar{F}_{\mathrm{d}}\right|$ per trial, (2) the mean absolute interaction torque $\left|\bar{T}_{\mathrm{d}}\right|$ per trial and (3) the RMS error of the signal tracking $E_{\operatorname{Tr}}$ per trial, defined as

$$
E_{\operatorname{Tr}}=\sqrt{\sum_{i=1}^{S}\left(\beta_{i}-v_{i}\right)^{2} / s},
$$

where $v_{i}$ is the angle of the multisine signal at the $i$ th sample, $\beta_{i}$ the exoskeleton elbow angle at the $i$ th sample and $s$ the signal length in samples $(s=60,000)$. For the subjective metrics, the measures are (4) the comfort rating $\bar{C}$ that was acquired after each trial, (5) the motion-hindrance rating $\bar{M}$ acquired after each trial, (6) the group weighted NASA TLX workload rating $\bar{W}_{\mathrm{WL}}$ and (7-12) the group weighted ratings on the individual TLX scales (PD-FR). 
Also, in order to reveal the influence of the experimental factors, mainly the kinematic setting, on the combined human-exoskeleton system during movement (thus, within each trial), five additional objective measures were processed into angle bins, per experiment. The binned dependent variables are (13) the raw, signed interaction force $\bar{F}_{\mathrm{d} \beta}$, (14) the raw, signed interaction torque $\bar{T}_{\mathrm{d} \beta}$, (15) the voluntary range of motion $R_{\beta}$ derived from the exoskeleton elbow joint angle $\beta,(16)$ the displacement of the linear compensation joint $d_{\text {lin }}$ and (17) the displacement of the rotary compensation joint $\Theta_{\text {rot. }}$. The first variables, $\bar{F}_{\mathrm{d} \beta}, \bar{T}_{\mathrm{d} \beta}$, $d_{\text {lin }}$ and $\Theta_{\text {rot }}$ were accumulated for each trial into nine bins that each spanned $10^{\circ}$ wide over the exoskeleton elbow joint angle $\beta$, in the range from $0^{\circ}$ to $90^{\circ}$. This span of the bins was chosen to have sufficient data points to derive the main trend of the data during movement while keeping a sufficiently good resolution. The voluntary range of motion $R_{\beta}$ is expressed in percent of multisine target angle $v$ reached. It is defined as the ratio $\left(s_{\beta} \times 100\right) / s_{v}$, with $s_{\beta}$ being the total number of samples for which $\beta$ lies inside a $10^{\circ}$-wide bin per trial and $s_{v}$ the total number of samples for which $v$ lies within the same $10^{\circ}$-wide bin. Thus, for this measure, the samples do not have to lie within the same bin at the same time. This calculus had to be done in order to arrive at an estimate of the available limb angle workspace, as the true human elbow joint angle $\alpha$ was not measured directly.

\subsubsection{Statistical analysis}

In order to analyse all aspects of the results, a series of twosided ANOVAs were performed on each dependent variable of the experiment.

First, the influence of the kinematic condition on the dependent variable was tested (test: $T 1$ ) by a two-way ANOVA considering the kinematic condition as main and the subjects as secondary factor, with six repetitions per subject $\left(T 1: n_{\mathrm{F} 1}=84, d f_{1}=1 ; n_{\mathrm{F} 2}=12, d f_{2}=13\right.$, rep. $\left.=6\right)$. Next, the influence of the pressure variation was tested $(T 2)$ as main, with subjects as secondary factor $\left(T 2: n_{\mathrm{F} 1}=\right.$ $28, d f_{1}=5 ; n_{\mathrm{F} 2}=12, d f_{2}=13$, rep. $\left.=2\right)$. Then (T3), the influence of the kinematic setting, was tested as main, with the influence of pressure variation as secondary factor $(T 3$ : $n_{\mathrm{F} 1}=84, d f_{1}=1 ; n_{\mathrm{F} 2}=28, d f_{2}=5$, rep. $\left.=14\right)$. This test allows investigation of the interaction between the main factors, with high statistical power.

In order to investigate differences between kinematic conditions only, excluding additional variance due to interface pressure variation (T4), six 2-factor ANOVAs were done for each pressure increment, with the kinematic configuration as main and again with subjects as secondary factor $\left(T 4: n_{\mathrm{F} 1}=14, d f_{1}=1 ; n_{\mathrm{F} 2}=2, d f_{2}=13\right.$, rep. $=$ 1). To test the pressure variation independent of the variance contributed by the kinematic levels (T5), two 2-way ANOVAs were performed for each dependent variable, with pressure condition as primary and subjects as secondary factor $\left(T 5: n_{\mathrm{F} 1}=14, d f_{1}=5 ; n_{\mathrm{F} 2}=6, d f_{2}=13\right.$, rep. $\left.=1\right)$.
At last, one 2-way ANOVA was computed per subject (T6) with kinematic condition as main and pressure condition as secondary factor $\left(T 6: n_{\mathrm{F} 1}=6, d f_{1}=1 ; n_{\mathrm{F} 2}=2, d f_{2}=5\right.$, rep. =1). $T 6$ allowed determining the interaction effects between the first two factors for each subject.

To reveal effects of the kinematic setting on the binned measures during elbow motion, $T 1$ was used for each bin. With these six tests, the main effects of all factors as well as their interaction can be analysed. All testing and analysis was performed in MATLAB. In the Results section, only significant results at 5\%-level or higher are reported. In figures, the $p$ values are encoded according to $* p<.05$, $* * p<.01, * * * p<.001$.

\section{4. pHRI model}

\subsection{Model structure}

Figure 3 shows the basic structure of the 1-d.o.f. pHRI model. In order to analyse the effects of geometric alignment between the human limb centre of rotation $I_{C R_{h}}$ and the wearable robot axis of motion $\mathrm{CR}_{\mathrm{e}}$ on interaction force, the mathematical model relates the offsets $x$ and $y$ between the two axes to the resulting displacement $d_{\text {tot }}$ of the exoskeleton attachment at the forearm. The total displacement $d_{\text {tot }}$ is created by a combination of linear $d_{\text {lin }}$ (Figure 3(a)) and rotary $d_{\text {rot }}$ (Figure 3(b)) terms. The offsets $x$ and $y$ are described in a fixed coordinate frame attached to the upper arm. Their direction is defined for a fully extended human limb in the rightmost illustrations of Figures $3(a)$ and (b).

The model considers the limb angle of rotation $\alpha(\alpha$ equals zero for the fully extended limb), or alternatively the robot's joint angle of rotation $\beta$, as well as the DenavitHartenberg (DH) parameters of the robot, the link length $l_{\text {ex }}$ and the link offset $z_{\text {ex }} . \beta_{0}$ is the resting angle of the robot if the human limb is fully extended (if $\alpha=0$ ). The rotary offset between human limb and robot link is described by $\gamma$. The model has been proposed and validated for parameter estimation and pHRI prediction in Schiele (2008a).

However, we included the two main output equations for $d_{\text {tot }}$ resolved for $\alpha$ and $\beta$ in the appendix. We consider model dependence from $\beta$ in this paper, since this is the variable that we could actually measure during the experiment. In Schiele (2008b), we show outputs of the model in dependence of the pure elbow rotation $\alpha$.

The force creation during motion is a linear approximation depending on the displacement output of the model according to

$$
F_{\mathrm{d}}=k \cdot d_{\mathrm{tot}}\left(\beta, x, y, l_{\mathrm{ex}}, z_{\mathrm{ex}}\right),
$$

where $k$ is the lumped stiffness between the human and the robot.

Different displacement-force relationships can be assumed; however, we showed (Schiele 2008a) that a simple 

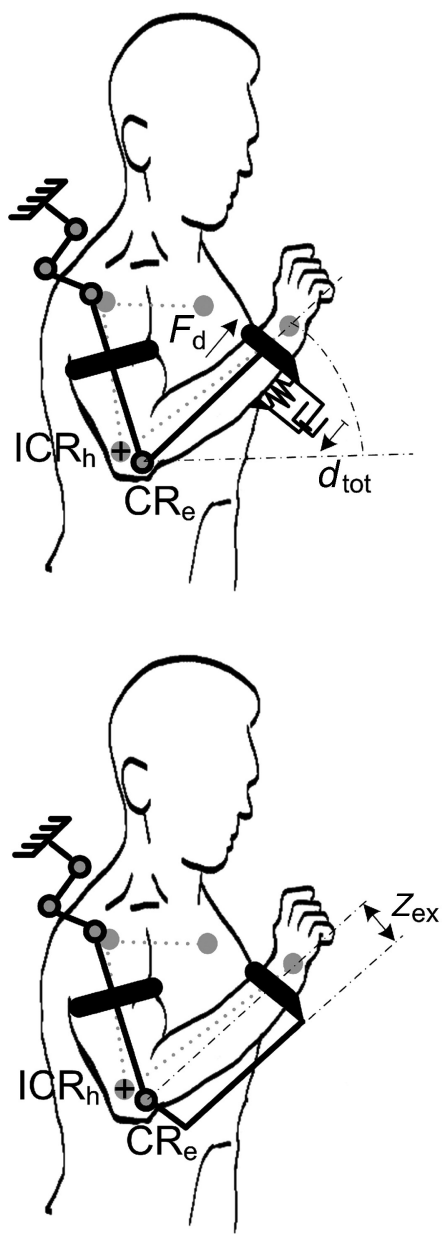

(a)

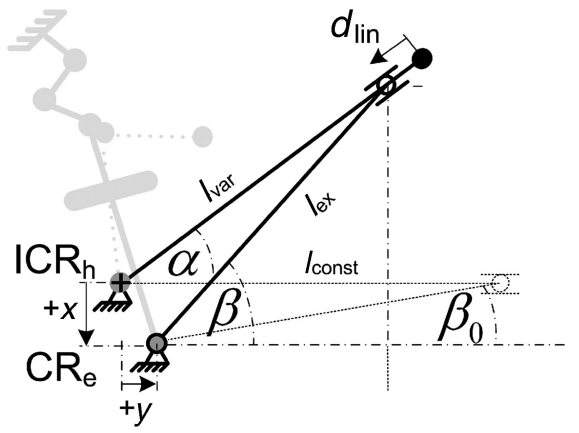

(b)

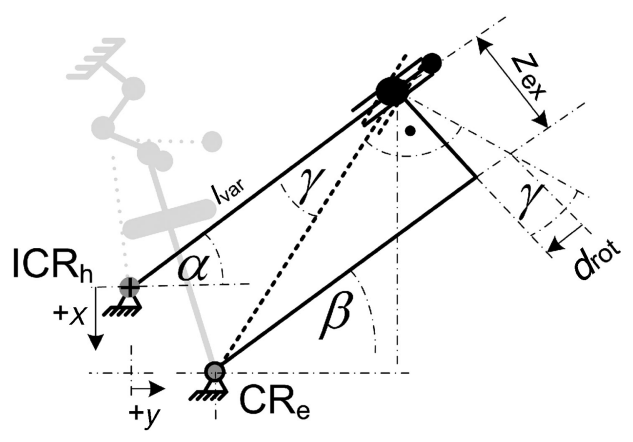

Figure 3. Graphical illustration of the main parameters of the 1-d.o.f. pHRI model that describes the creation of the interaction force $F_{\mathrm{d}}$ depending on offsets $x$ and $y$ between the human elbow centre of rotation $\left(\mathrm{ICR}_{\mathrm{h}}\right)$ and the wearable robot centre of rotation $\left(\mathrm{CR}_{\mathrm{e}}\right)$. The offsets are described in the upper-arm coordinate system for a fully extended limb. The Denavit-Hartenberg parameters of the robot $l_{\text {ex }}$ (link length) and $z_{\text {ex }}$ (link offset) are inputs to the model. The angle of the human limb is $\alpha$, whereas the angle of the exoskeleton joint is $\beta$. In (a), the linear displacement contribution $d_{\text {lin }}$ is depicted. $\beta_{0}$ describes the rest position of the robot during the fully extended human limb. In (b), the displacement contribution $d_{\text {rot }}$ stemming from rotary offsets $\gamma$ and the robot link offset is illustrated.

linear relationship between displacement and force suffices to describe measured forces accurately with the model.

\subsection{Model parameter estimation}

The binned measured force $\bar{F}_{\mathrm{d} \beta}$ acquired during the $L$ trials was used as input to the parameter estimation with the pHRI model for each subject and the subject group. This way the geometric model parameters $x, y, l_{\mathrm{ex}}$ and $z_{\mathrm{ex}}$, as well as the attachment stiffness $k$ could be estimated from the measured data. To identify the parameters for the subject group, the mean forces $\bar{F}_{\mathrm{d} \beta}$ of all 14 subjects were used. For the individual subjects, mean forces $\overline{\bar{F}}_{\mathrm{d} \beta}$ over all the six $L$ trials were used. The parameter estimation was performed by means of non-linear least-squares optimisation as described in Schiele (2008a). Goodness of fit was determined by the coefficient of determination $R^{2}$, the norm of the residuals |res.| and graphical residual plots.

\section{Results}

\subsection{Interaction force prediction from pHRI model}

Figure 4 shows the predicted outputs of the pHRI model for interaction forces $F_{\mathrm{dm}}$ depending on combination of offsets $x$ and $y$ over the robotic limb model angle $\beta_{\mathrm{m}}$.

Influence of the link parameter $z_{\text {ex }}$ on the force is shown for a range of values from 0 to $0.14 \mathrm{~m}$. The attachment stiffness was taken from Schiele (2008a) to be $200 \mathrm{~N} / \mathrm{m}$ and the link length $l_{\text {ex }}$ was set to $0.167 \mathrm{~m}$, which is the true value for the EXARM exoskeleton in the $L$ configuration. For all graphs shown in Figure 4, $x$ was like $y$, equal to $0.05 \mathrm{~m}$, and the directions of the offsets are indicated atop each graph. This represents a typical example. It can be seen that the interaction force shows very different characteristics over the workspace, depending on the directional combinations of offsets between $\mathrm{ICR}_{\mathrm{h}}$ and $\mathrm{CR}_{\mathrm{e}}$. Here, predicted peak forces are in the order of $\pm 30 \mathrm{~N}$. 

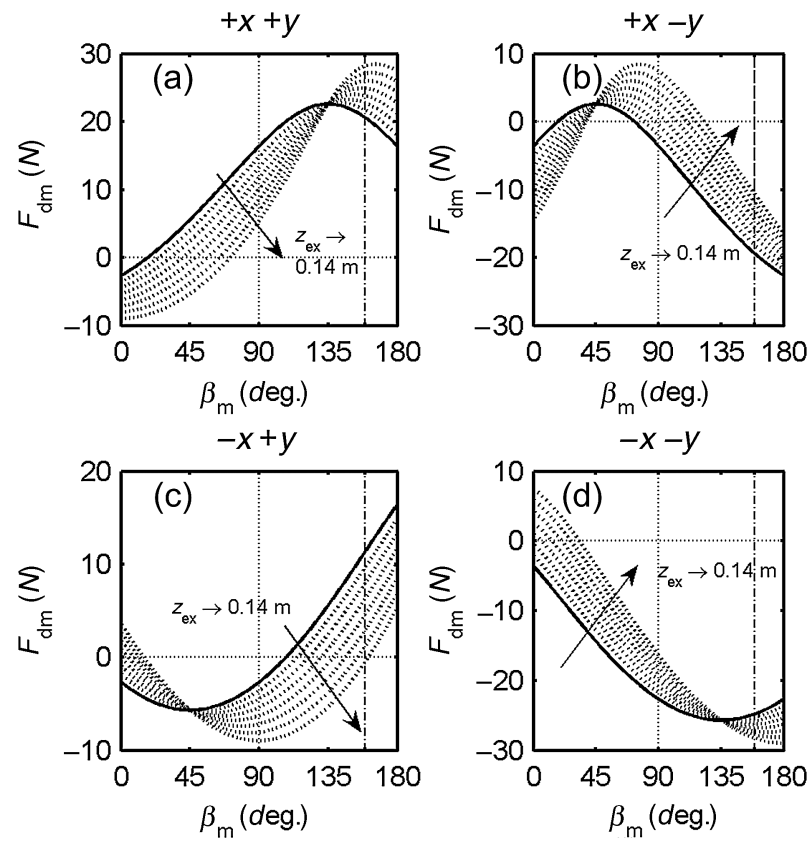

Figure 4. Exemplary model output from the 1-d.o.f. pHRI model. Interaction force $F_{\mathrm{d}}$ experienced between the human arm and the robot attachment is depicted over the angle $\beta_{\mathrm{m}}$ of a wearable robot model, for different link offsets $z_{\text {ex }}$ ranging from 0 to $0.14 \mathrm{~m}\left(z_{\mathrm{ex}}=\right.$ 0 on base of indicated arrow/thick line). The force is displayed for $|x|=|y|=0.05 \mathrm{~m}$, with the sign of the offset indicated on top of each graph. Dashed lines indicate the maximum elbow rotation angle for the 95th percentile of US male population.

\subsection{Measured interaction forces and torques}

The measures from the experiment will show typical magnitudes of typical interface loads. The full spectrum of measured raw peak-to-peak interaction forces $F_{\mathrm{d}}$ span for the group from -232 to $165 \mathrm{~N}$ for the $L$ and from -57 to $70 \mathrm{~N}$ for the $U$ condition, as shown in Figure 5(a). The full spectrum of measured raw peak-to-peak interaction torques ()$_{d}$ for the group span from -1.0 to $1.46 \mathrm{Nm}$ for $L$ and from -0.4 to $0.60 \mathrm{Nm}$ for the $U$ kinematic condition, as shown in Figure 5(d).

The mean loads $\left|\bar{F}_{\mathrm{d}}\right|$ and $\left|\bar{T}_{\mathrm{d}}\right|$ over all experiment trials and subjects are shown as boxplots in Figure 1(b) and (e), and show differences over the kinematic setting levels $L$ (locked) and $U$ (unlocked passive joints). Bar graphs in Figure 5(c) and (f) show the group means of the interaction loads over attachment pressure for both kinematic levels. Error bars indicate their $95 \%$ confidence interval (CI) on correct estimate of the means.

The mean interaction force per trial $\left|\bar{F}_{\mathrm{d}}\right|$ is significantly lower in the $U$ condition $(14.02 \pm 11.95 \mathrm{~N})$ than in the $L$ condition $(17.75 \pm 16.33 \mathrm{~N})$ for the entire subject group $(p<.05)$. The subjects contribute heavily to the measured variance $(p<.001)$, which is apparent in the large spread of the data presented in Figure 5(b). The kinematic setting factor shows strong interaction with the subject factor $(p<$ $.001)$.

The mean torque per trial $\left|\bar{T}_{\mathrm{d}}\right|$ is also significantly lower in the $U$ condition $(0.109 \pm 0.099 \mathrm{Nm})$ than in the $L$
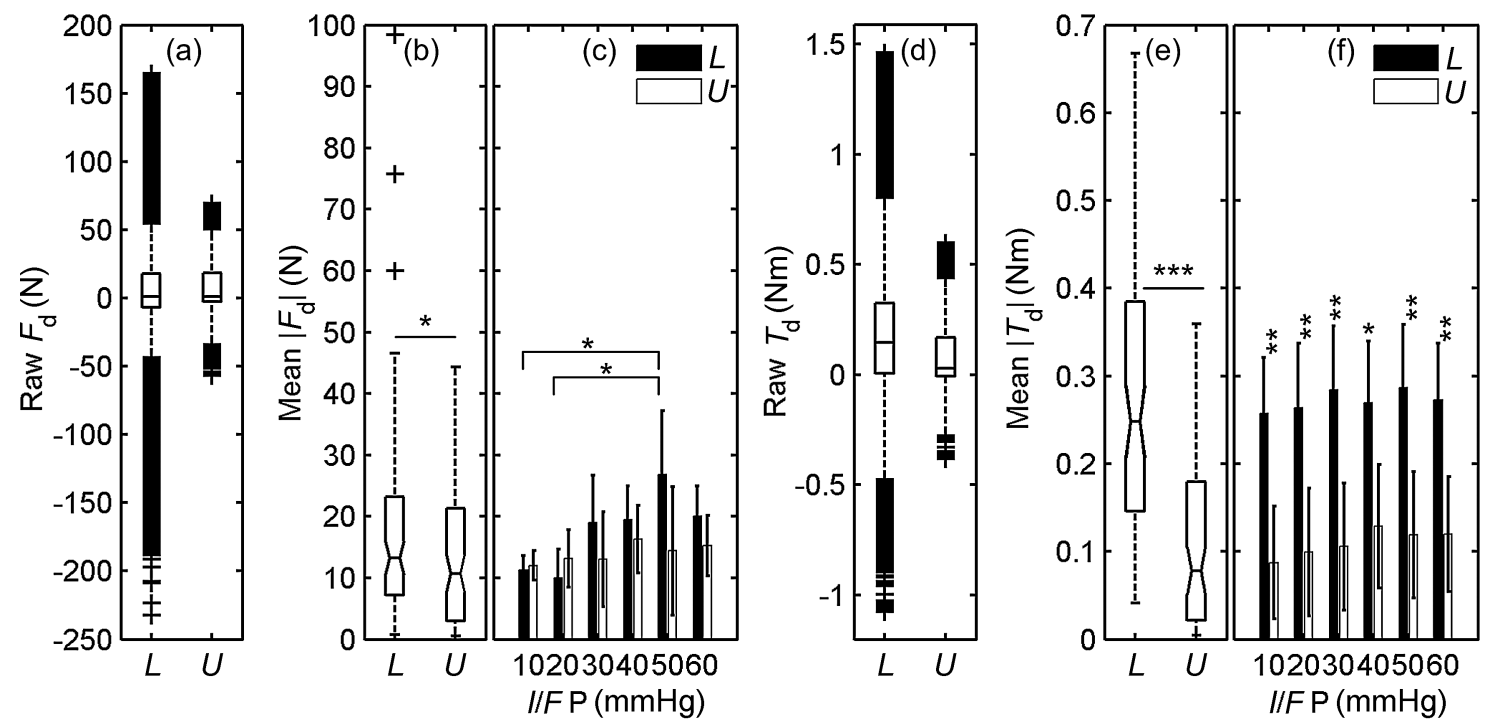

Figure 5. (a) and (d) show the raw measured force $F_{\mathrm{d}}$ and torque $T_{\mathrm{d}}$ over all experiments as boxplots with outliers (indicated by + markers). (b) and (c) show the mean absolute interaction force $\left|\vec{F}_{\mathrm{d}}\right|$. (b) shows boxplots of the force over the two levels of the kinematic setting factor, with the full measured variance $(n=84$ per level). This data was used for the two-way ANOVA test $T 1$. (c) shows the mean force values that are output from six two-way ANOVAs (T3) that compared means between kinematic settings per pressure increment. The $95 \%$ CI of the mean computed for $T 3$ is displayed by error bars. The variance due to subjects is removed by the ANOVA. Test $T 4$ compared the means between pressure increments of equal kinematic level. (e) and (f) show the mean absolute interaction torque $\left|\bar{T}_{\mathrm{d}}\right|$ in the same way as (b) and (c) do for the force. Significance levels are coded as $* p<.05,{ }^{* *} p<.01,{ }^{* * *} p<.001$. 

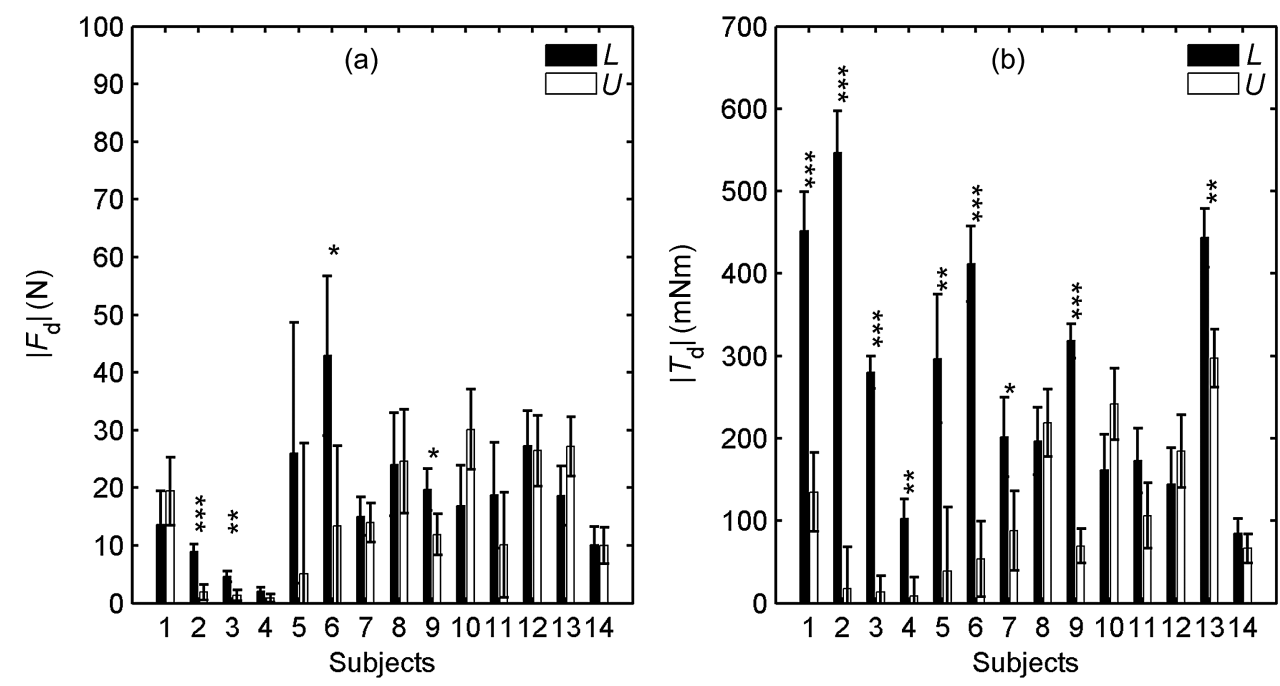

Figure 6. Mean absolute interaction force $\left|\bar{F}_{\mathrm{d}}\right|$ (a) and mean absolute interaction torque $\left|\bar{T}_{\mathrm{d}}\right|$ (b) exerted per subject over both kinematic setting levels $L$ (locked) and $U$ (unlocked). Each bar shows the mean loads per experiment per condition $(n=6)$. The error bars indicate the $95 \%$ confidence interval of the means, which are computed by two-way ANOVA $(T 5)$. Main factor analysed is the kinematic configuration $(L, U)$, secondary factor is the interface pressure. While significant differences exist for the kinematic setting factor, no differences exist between the pressure levels. Significant differences for the kinematic setting are encoded as $*<<.05,{ }^{* *} p<.01, * * * p<.001$.

condition $(0.272 \pm 0.153 \mathrm{Nm})$ for the subject group $(p<$ $.001)$. Also, the subjects contribute significantly to the measured variance $(p<.001)$ and the kinematic setting again shows interaction with the subjects $(p<.001)$. At all six pressure increments, the interaction torque is smaller for the $U$ condition than for the $L$ condition, as can be seen in Figure 5(f).

The effect of the kinematic setting on the interaction loads per subject is shown in Figure 6. Depicted are the mean values of the load for each kinematic condition. The $95 \% \mathrm{CI}$ is shown again by the whiskers. The statistical testing reveals that four subjects experience significantly less force in the $U$ condition (Figure 6(a)) $\left(p_{\mathrm{S} 2}<.001\right.$, $\left.p_{\mathrm{S} 3}<.01, p_{\mathrm{S} 6}<.05, p_{\mathrm{S} 9}<.05\right)$.

Interestingly, no subject experiences significantly less force in the $L$ condition. With respect to the interaction torque (Figure 6(b)), the effect is even more pronounced, with nine subjects experiencing significantly less interaction torque in the $U$ kinematic condition and no subject that experiences less torque in the $L$ condition $\left(p_{\mathrm{S} 1}<.001\right.$, $p_{\mathrm{S} 2}<.001, p_{\mathrm{S} 3}<.001, p_{\mathrm{S} 4}<.01, p_{\mathrm{S} 5}<.01, p_{\mathrm{S} 6}<.001$, $\left.p_{\mathrm{S} 7}<.05, p_{\mathrm{S} 9}<.001, p_{\mathrm{S} 13}<.001\right)$.

The binned interaction forces $\bar{F}_{\mathrm{d} \beta}$ and torques $\bar{T}_{\mathrm{d} \beta}$ of the subject group are depicted in Figure 7 for the exoskeleton elbow rotation angle $\beta$. The standard deviation is shown as upper and lower bounds on each curve. The mean force exertion $\bar{F}_{\mathrm{d} \beta}$ (Figure 7(a)) is significantly smaller for the $U$ kinematic setting in the $85^{\circ}$ angle bin $(p<.01)$. The exerted interaction torque $\bar{T}_{\mathrm{d} \beta}$ over the workspace (Figure 7(b)) is smaller in the $U$ kinematic condition for the first six bins, spanning from $0^{\circ}$ to $60^{\circ}(p<.001, p<.001, p<$
$.001, p<.001, p<.001, p<.05)$. Also here, for both dependent variables, the two factors kinematic setting and subjects show a strong interaction term for each bin $(p<$ $.001)$.

\subsection{Identification of pHRI model parameters from measured interaction forces in $L$ condition}

The offset estimates $\hat{x}$ and $\hat{y}$, as well as the estimated attachment stiffness $\hat{k}$, are shown per subject in Table 1 .

The table also shows the coefficient of determination $R^{2}$ and the norm of the residuals |res.| which indicate the goodness of fit between the identified pHRI model and the measured $\bar{F}_{\mathrm{d} \beta}$.

Graphs of the model fits for each subject are depicted in Figure 8, along with the raw force values in $2^{\circ}$-wide bins over the exoskeleton elbow angle. The graphs are sorted according to the direction of the offsets similar to Figure 4 to show the capability of the model to identify the measured force characteristic in the $L$ condition.

The model parameters identified from the mean force measures $\bar{F}_{\mathrm{d} \beta}$ for the entire group are as follows:

$$
\begin{aligned}
& \hat{k}_{\mathrm{G}}=3.115 \mathrm{~N} / \mathrm{m}, \quad \hat{x}_{\mathrm{G}}=0.0076 \mathrm{~m}, \quad \hat{y}_{\mathrm{G}}=-0.097 \mathrm{~m}, \\
& \hat{l}_{\mathrm{exG}}=0.167 \mathrm{~m}, \quad \hat{z}_{\mathrm{exG}}=0.13 \mathrm{~m} .
\end{aligned}
$$

The output graph of the pHRI model with those parameters is displayed for the group measures in Figure 7(a) $\left(R^{2}=97.53 \%, \mid\right.$ res. $\left.\mid=3.45 \mathrm{~N}\right)$. 

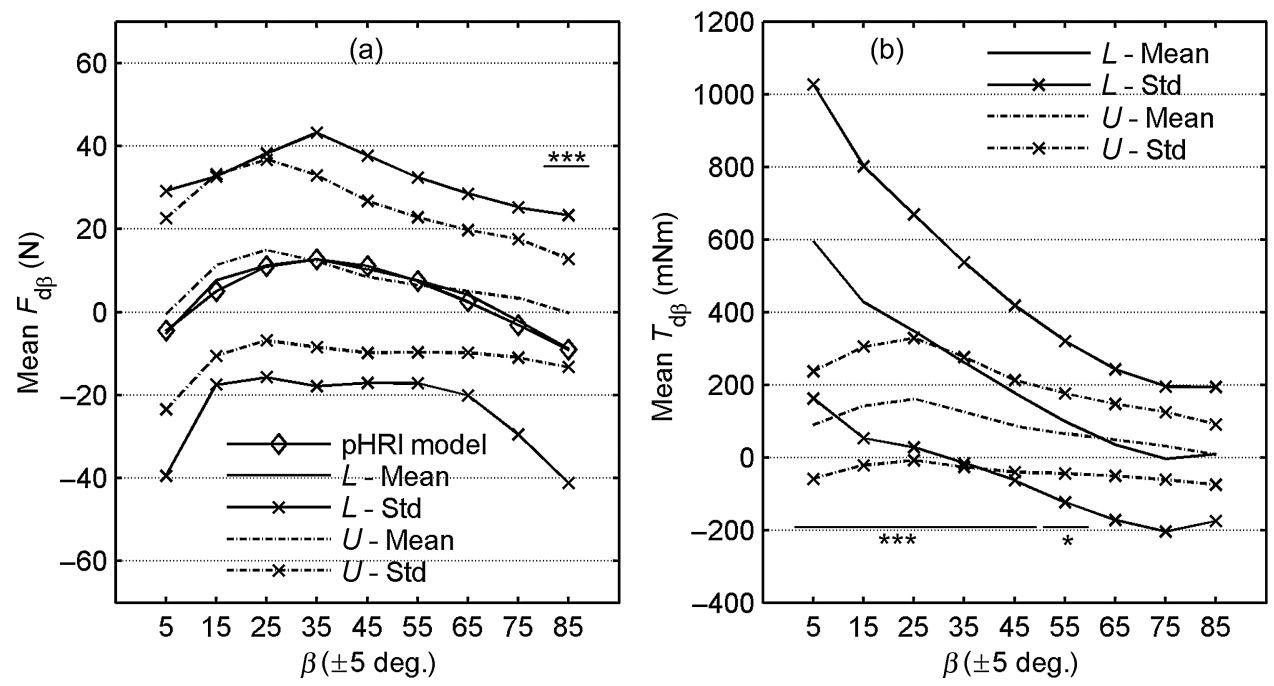

Figure 7. Binned interaction force output measure $\bar{F}_{\mathrm{d} \beta}$ and output torque $\bar{T}_{\mathrm{d} \beta}$ exerted during motion of the elbow joint of the exoskeleton. In (a) the mean force exerted by all subjects and all experiments over the exoskeleton elbow angle is shown together with the full measured standard deviation for both levels of the kinematic setting factor ( $L$ and $U$ ). The output of the pHRI model with identified parameters from the $L$ force data is shown in addition. Bins indicated by asterisk feature significantly different mean load between the two kinematic settings. Significance tests were performed on each bin by two-way ANOVA (T6), for which the kinematic setting was the first and the subjects were the second factor; thus, the variance due to subjects that is shown in the figure was removed by the test. In (b), equivalent outputs are shown for the mean interaction torque. Significance levels are coded as $* p<.05, * * * p<.001$.

\subsection{Signal-tracking performance}

The RMS-tracking error $E_{\mathrm{Tr}}$ for the subject group, over all trials, varies within $13.27 \pm 2.8^{\circ}$. A boxplot in Figure 9(a) shows the entire spread of $E_{\operatorname{Tr}}$ measurement points. The subjects contribute significantly to the variability of the RMS-tracking error measures as expected due to capability differences $(p<.001)$. It can be seen from Figure 9(b)

Table 1. Extract of identified pHRI-model parameters from measured interaction forces (per subject).

\begin{tabular}{lrrrrr}
\hline Subject & $\hat{k}(\mathrm{~N} / \mathrm{m})$ & $\hat{x}\left(-10^{-3} \mathrm{~m}\right)$ & $\hat{y}\left(-10^{-3} \mathrm{~m}\right)$ & $R^{2}(\%)$ & $\mid$ res.| $(\mathrm{N})$ \\
\hline 1 & 251 & -22.3 & -78.9 & 89.2 & 17.7 \\
2 & 249 & 68.4 & 18.6 & 98.9 & 5.2 \\
3 & 222 & 48.2 & 58.5 & 97.3 & 5.1 \\
4 & 249 & 2.1 & 2.1 & 21.6 & 6.3 \\
5 & 256 & 124.4 & -48.7 & 93.5 & 23.0 \\
6 & 286 & 136.2 & 7.9 & 83.8 & 47.2 \\
7 & 264 & -17.2 & -116.9 & 99.2 & 8.3 \\
8 & 374 & 3.5 & -87.7 & 98.6 & 11.2 \\
9 & 237 & 78.3 & 10.3 & 66.4 & 26.0 \\
10 & 250 & 1.8 & -76.6 & 91.6 & 16.3 \\
11 & 253 & -15.0 & -54.2 & 56.2 & 42.7 \\
12 & 271 & 5.6 & -77.1 & 94.5 & 15.9 \\
13 & 373 & -0.5 & -81.3 & 98.4 & 8.1 \\
14 & 284 & 25.2 & -115.6 & 95.7 & 10.1 \\
\hline
\end{tabular}

Note: Results of the pHRI model parameter identification $(\hat{k}$ : estimated attachment stiffness; $\hat{x}$ : estimated offset of exoskeleton joint in $x$ direction; $\hat{y}$ : estimated offset of exoskeleton joint in $y$ direction; $R^{2}$ : coefficient of determination; |res.|: norm of residual errors). that in the range from 10 to $30 \mathrm{mmHg}$, two measures are lower for the $U$ condition, while in the range from 40 to 60 $\mathrm{mmHg}$, two measures are higher for the $U$ condition. Two post-hoc tests on the effect of kinematic setting over the two pressure ranges confirmed that in the range from 10 to $30 \mathrm{mmHg}$ subjects perform better in the $U$ configuration $(p<.001)$, whereas in the range from 40 to $60 \mathrm{mmHg}$ they perform better in the $L$ configuration $(p<.001)$.

\subsection{Voluntary range of motion}

In Figure 10(a), a full spectrum of the target and tracking signals is shown. It can be seen how often $\left(s_{v}\right)$ the multisine target signal $v$ and the subject's tracking signals in $L$ or $U$ kinematic configuration $\left(s_{\beta U}\right.$ and $\left.s_{\beta L}\right)$ resided within one of the nine bins of elbow workspace $\alpha$ during the experiments (range $R(v)=R(\alpha)$ ). In Figure 10(b), the group mean of the voluntary range of motion $R_{\beta}$ for both kinematic configurations is shown as percent of the target signal $v$ over the workspace.

It can be seen that on both stroke-ends, the subjects reached the target signal substantially less than $100 \%$. While in the range of $0^{\circ}-10^{\circ}$, the subjects reached the target signal equally often for the two kinematic configurations, there is a significant difference at the $80^{\circ}-90^{\circ}$ stroke-end.

Subjects reached the far stroke-end about $20 \%$ more often $(83.4 \%$ vs. $68.2 \%)$ in the $U$ kinematic configuration than in the $L$ configuration $(p<.001)$. The kinematic setting affects the range of motion also in other parts of the 

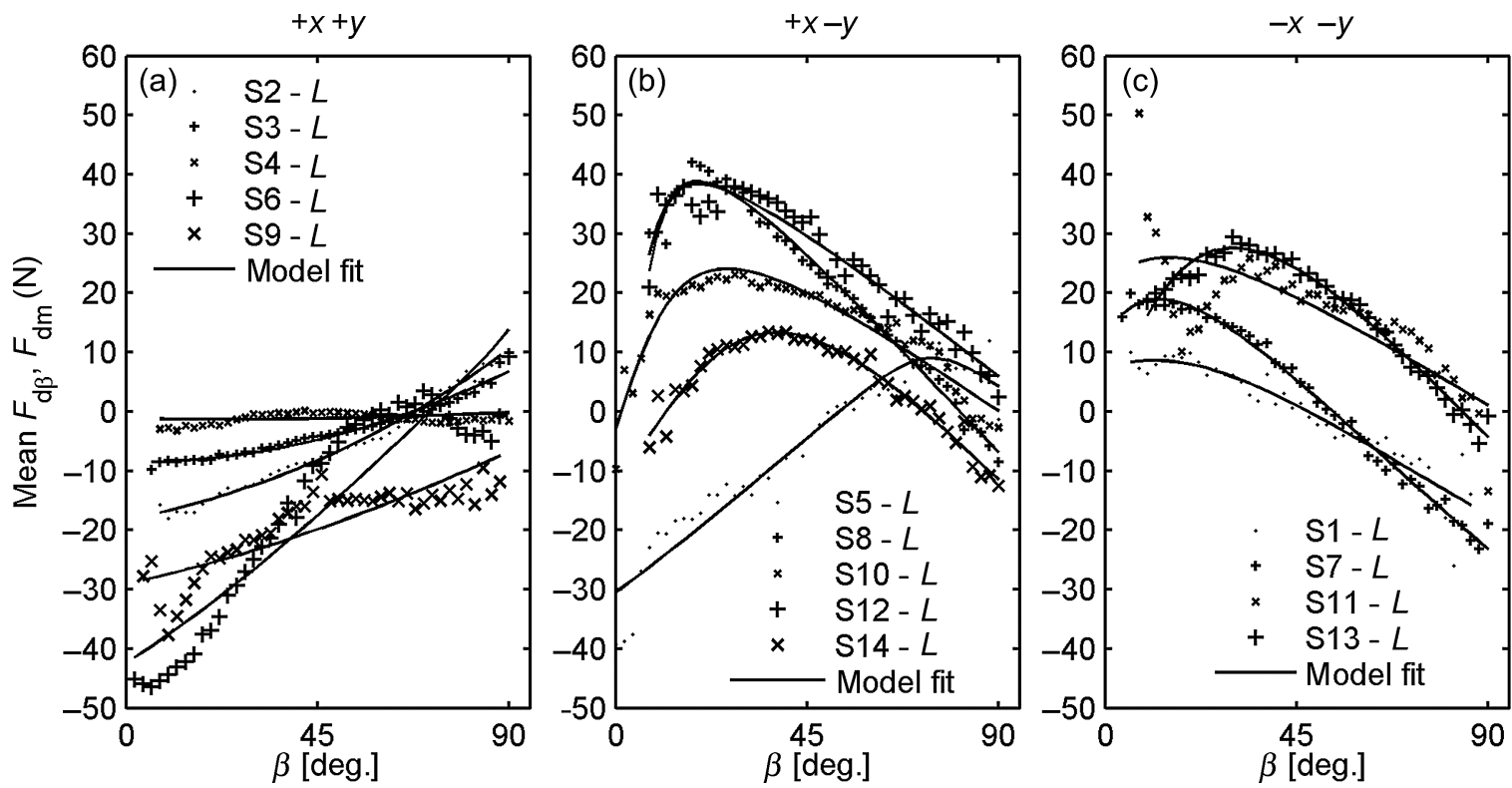

Figure 8. Raw force measurements $F_{\mathrm{d}}$ of the subjects (S) over the exoskeleton angle $\beta$ per six trials. The model fitted force characteristic $F_{\mathrm{dm}}$ is shown per subject on the same graphs. All measured forces are acquired during the $L$ exoskeleton trials (six per subject) and averaged within $2^{\circ}$-wide angle bins. The illustrations are sorted for identified offsets from $+x,+y(\mathrm{a}),+x,-y$ (b) and $-x,-y$ (c). There are no subjects that had $-x,+y$ offsets in this experiment.
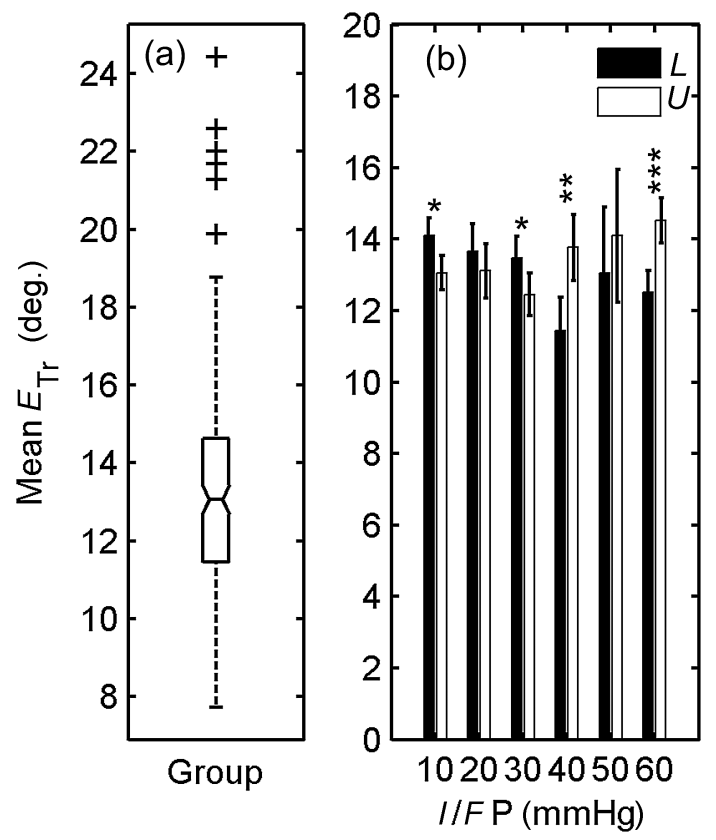

Figure 9. Boxplot (a) of overall signal-tracking error $E_{\operatorname{Tr}}$ of the subject group. In (b), mean values of $E_{\mathrm{Tr}}$ of the group are shown over kinematic condition and attachment pressure levels. The $95 \%$ CI that is shown results $T 3(* p<.05, * * p<.01, * * * p<.001)$.
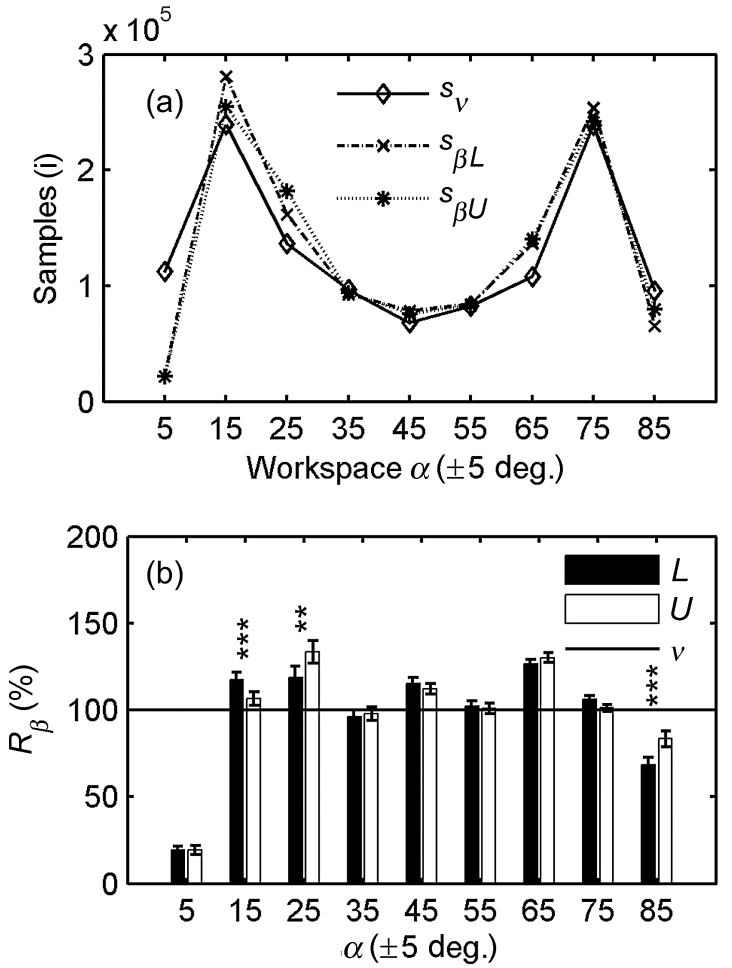

Figure 10. (a) Range of motion available with exoskeleton in both kinematic configurations. Total number of samples $i$ for which the target multisine signal $v$ and the tracking signals in $L$ and $U$ kinematic settings reside within the same nine $10^{\circ}$-wide bins of the elbow workspace. (b) The voluntary range of motion $R_{\beta}$ for both kinematic settings in $\%$ of input signal reached over the workspace $(* p<.05, * * p<.01, * * * p<.001)$. 
workspace; however, there subjects can all reach $100 \%$ of the tracking signal in both conditions. Only the overshoot over the target signal is affected and the differences are less profound $(p<.001, p<.01)$.

\subsection{Motion of passive compensatory joints}

The linear passive compensation joint $d_{\text {lin }}$ of the exoskeleton operated within a range of $0-35 \mathrm{~mm}$ in the $U$ trials. The rotary compensation joint $\Theta_{\text {rot }}$ operated within a range of $-11^{\circ}$ to $+25^{\circ}$. Figure 11 depicts the mean motion of $d_{\text {lin }}$ in (a) and of $\Theta_{\text {rot }}$ in (b), over all trials and subjects.

\subsection{Preferred attachment pressure of the subject group}

When asked after the experiments, the subject group selected their preferred 'most comfortable' attachment pressures to be within $21.6 \pm 8.7 \mathrm{mmHg}$ for the upper-arm air-cuff and to be within $20.1 \pm 7.7 \mathrm{mmHg}$ for the forearm air-cuff.

\subsection{Subjective metric I: comfort questionnaire}

The interface pressure has an influence on the perceived comfort of the subjects. At $60 \mathrm{mmHg}$, the perceived comfort was lower than at all other pressure increments. In particular, the perceived comfort at $60 \mathrm{mmHg}$ was lower than at $50 \mathrm{mmHg}(p<.01)$ and with a more profound effect lower than at $10 \mathrm{mmHg}(p<.001)$. Figure 12 depicts the measured $\bar{C}$ ratings for the group over the original scale.

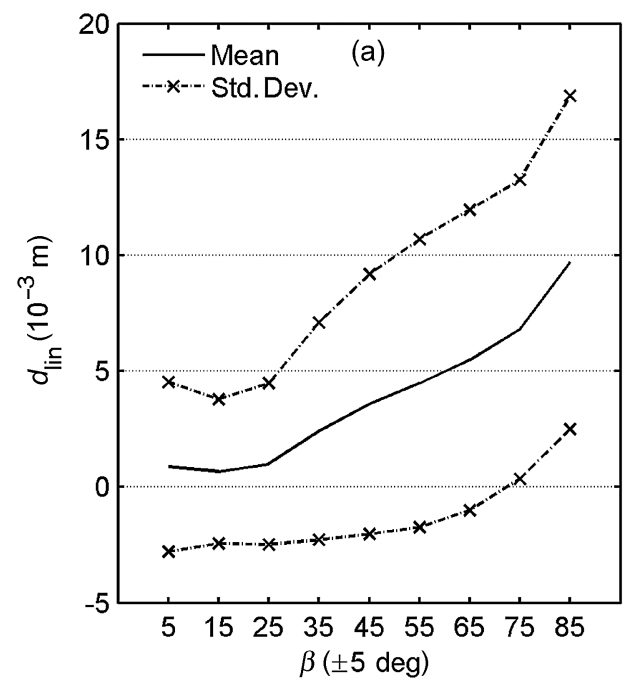

Testing for influence of the kinematic setting over specific pressures revealed that at $30 \mathrm{mmHg}$, the subjects rated the perceived comfort higher in the $U$ configuration than in the $L$ configuration $(p<.05)$. The highest mean comfort rating of the group was given for $20 \mathrm{mmHg}$ in the $U$ condition (Figure 12(b)).

\subsection{Subjective metric II: motion hindrance questionnaire}

The subjects did not rate the motion hindrance differently between the kinematic or the pressure factors of the experiment.

\subsection{Subjective metric III: NASA TLX ratings}

The following mean group weighting factors were determined after the experiment for the NASA TLX rating scales: mental demand (MD), 1.93; physical demand (PD), 2.21; temporal demand (TD), 3.0; performance (OP), 2.71; effort (EF), 2.79; frustration (FR), 2.36.

Analysis of the total perceived $\bar{W}_{W L}$ scores did not reveal effects from the kinematic or pressure variation on workload. The effect of kinematic variation on the individual rating scales is shown in Figure 13(a), while the effect of pressure variation on the six individual rating scales is illustrated in Figure 13(b). The kinematic setting has an influence on the mental demand rating. In particular, the subjects experience less mental demand in the $U$ condition than in the $L$ condition $(p<.05)$.

The pressure settings showed influence on the ratings of the subjects on the individual TLX scales. Perceived mental demand decreases towards higher pressures, with mental

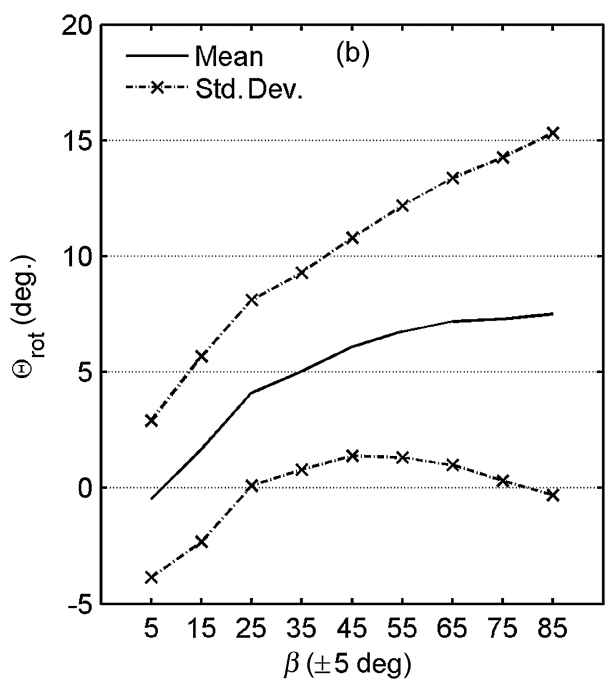

Figure 11. Mean motion caused in the passive compensation joints during trials with the $U$ kinematic setting. In (a) motion of the linear compensation joint $d_{\text {lin }}$ is shown over the exoskeleton elbow rotation angle $\beta$. In (b) motion of the rotary compensation joint $\Theta_{\text {rot }}$ is also depicted over the exoskeleton elbow angle $\beta$. By their movement, the joints lower the perceived interaction force stemming from misalignment of the axes of rotation between exoskeleton and human limb. 

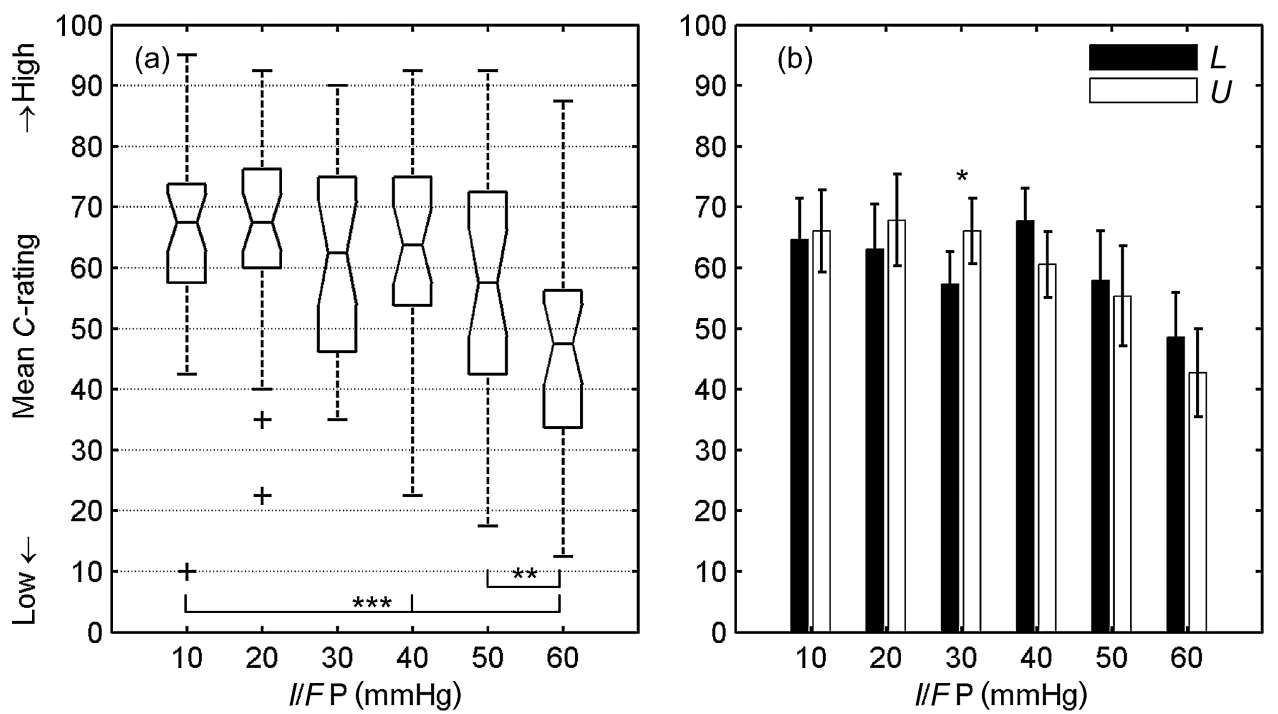

Figure 12. Mean comfort rating $\bar{C}$ of the subject group is shown as boxplots in (a) over the six different attachment pressure increments. The means between the groups were compared by $T 2$. Significant differences are coded by asterisk. In (b), the mean comfort ratings of the group are shown over attachment pressure and kinematic setting. The $95 \%$ CI shown results from comparison of the kinematic setting factors at each pressure increment $T 3$. Significant differences between means are indicated per pressure increment by asterisk $(* p<.05$, $* * p<.01, * * * p<.001)$.

load experienced at $60 \mathrm{mmHg}$ being lower than at 10,30 and $50 \mathrm{mmHg}(p<.05, p<.01, p<.05)$. Physical demand ratings are influenced by the pressure factor as well. In particular, physical demand shows an increasing trend with higher pressures. Again, post-hoc tests confirmed higher physical demand ratings for $60 \mathrm{mmHg}$ than for 10, 20 and $40 \mathrm{mmHg}(p<.01, p<.005, p<.01)$.
The subject's rating on the effort scale showed influence to the pressure variation. In particular, subjects rated higher effort at higher interface pressures. The post-hoc tests revealed that at $50 \mathrm{mmHg}$, the subjects rated effort higher than at $10 \mathrm{mmHg}$, and at $60 \mathrm{mmHg}$ they rated effort higher than at 10 and $20 \mathrm{mmHg}(p<.01, p<.001$, $p<.01)$.
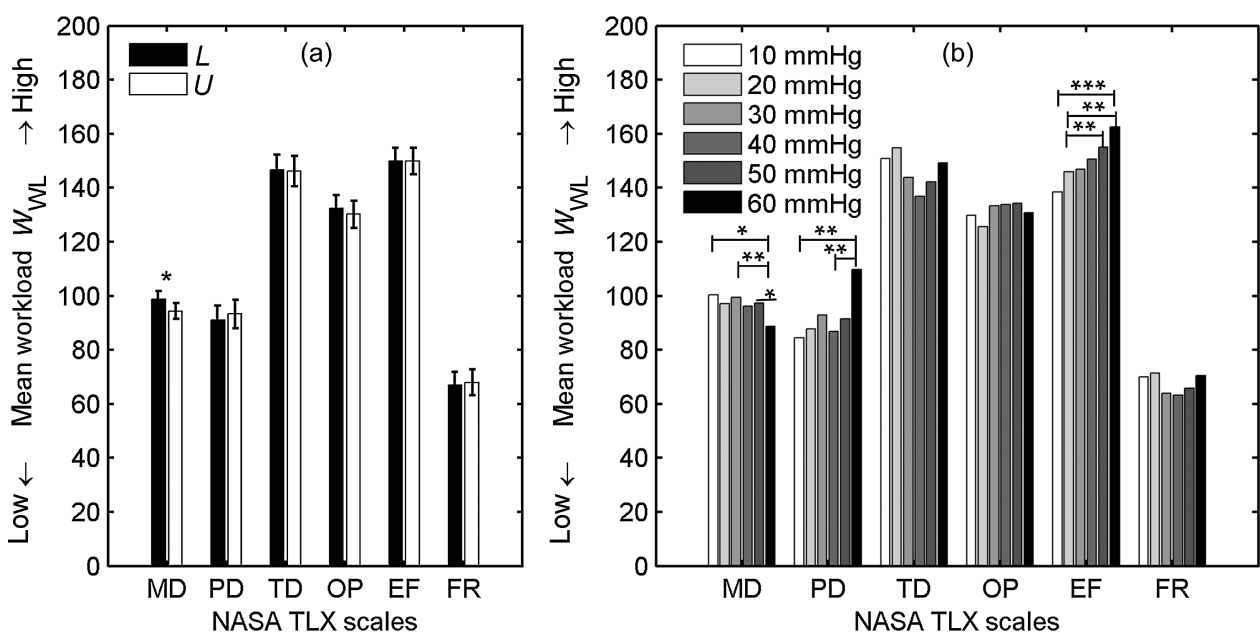

Figure 13. Results of the subject group weighted workload ratings $\bar{W}_{\mathrm{WL}}$ for the individual NASA TLX scales (MD: Mental Demand, PD: Physical Demand, TD: Temporal Demand, OP: Operator Performance, EF: Effort, FR: Frustration Level). In (a), influence of the kinematic setting on the TLX scales is depicted. Differences between $L$ and $U$ were tested with $T 1$. The $95 \%$ CI on the difference between the means is shown as computed by ANOVA analysis. In (b), mean workload ratings are shown per TLX scale for each attachment pressure settings, ranging from 10 to $60 \mathrm{mmHg}$. Significant differences are shown by asterisk. Significance levels are coded as $* p<.05$, $* * p<.01$, $* * * p<.001$. 


\section{Discussion}

From the results presented above, it is evident that a wearable robot structure that includes passive compensation joints is better.

It can be seen from Figures 5 and 6 that the $U$ kinematic condition causes significantly less interaction load between the exoskeleton and the human arm. Peak load (Figures 5(a) and (d)) at the human-machine interface of up to $232 \mathrm{~N}$ and $1.46 \mathrm{Nm}$ were created for experiments with $L$ passive joints, whereas in the $U$ condition, the additional passive joints reduced those peak forces by $70 \%$ to $70 \mathrm{~N}$ and the torques by $60 \%$ to only about $0.6 \mathrm{Nm}$.

From Figure 7 it can be seen that the exoskeleton in the $U$ configuration reduces the interaction forces most significantly in the motion end ranges. Here, the peak loads are created during movement in the $L$ configuration. The passive joints of the exoskeleton significantly reduce the force in the far stroke-end (Figure 7(a)) and the torque in the near stroke-end (Figure 7(b)). Figure 11(a) shows that the linear compensation joint starts moving at about the same angle in workspace $\left(\sim 25^{\circ}\right)$ where the mean interaction force of the group starts to peak. The linear joint moves more towards the far stroke-end. The mean force for the $U$ condition consequently has a smaller spread in this range and decreases towards 0 near the $90^{\circ}$ stroke-end. This is where the linear compensation joint experiences most stroke (Figure 11(a)). From the narrower range of forces experienced in the $U$ condition (Figure 7), it can be seen that the linear joint works to remove peak forces. This offers an explanation for the differences seen between the two kinematic conditions in Figure 5(a), where the $U$ setting does not contain nearly as large forces as the $L$ setting does. The mean force in the $U$ condition decreases faster over the working range than the mean force measured with the exoskeleton in $L$ kinematic condition (Figure 7(a)). However, as can be seen in the behaviour of the mean force in the $L$ trials in Figure 7(a), the levelling-off of the force is caused not only by the passive joints involved in the $U$ configuration, but also by the geometric properties of the attachment itself. The pHRI model fit shown in Figure 7(a) indicates that with the average offsets of the group of $+7.6 \mathrm{~cm}$ in the $x$ direction and $-9.7 \mathrm{~cm}$ in the $y$ direction, the force experienced by most subjects changes direction from distal to proximal at about $35^{\circ}$ and then starts to increase in proximal direction continuously. This trend can also be seen in Figures 8(b) and (c). The subjects featuring $+x /+y$ offsets create force in proximal direction near the extended limb position and have no turning point. The mostly negative forces near both stroke-ends explain the large negative peak forces that are shown in Figure 5(a) for the $L$ trials. The model prediction in Figure 4(b) shows that the trend of the force in the $L$ configuration for the group would continue to increase towards larger angles. Clearly, in a real application, angles above $90^{\circ}$ for the elbow are quite natural, and therefore a kinematic design with compensatory joints is better (where such forces are removed).

The individual interaction force measures shown in Figure 8 clarify how the force trend of the group is composed. It can be seen that the pHRI model is able to fit the various apparent trends.

For the group, we see in Figure 10 that the voluntary range of motion in the far stroke-end is more limited for the $L$ condition than for the $U$ condition. Subjects can reach closer to the target signal in the $85^{\circ}$-angle bin. They have about $20 \%$ more range of motion available in the $U$ condition. It appears that the voluntary range of motion is decreased by increasing interaction forces over large angles in the workspace. The $U$ kinematic setting lowers such forces by adjusting the passive compensatory joints (Figure 11), and thus allows a greater range of motion in the far stroke-end.

However, for both kinematic settings, the voluntary range of motion close to the fully extended $\left(0^{\circ}\right)$ strokeend is limited. This can also be explained. The average offset for the subject group in $x$ direction is positive $(+x)$, according to the sign convention of our model (Figure 3). For some subjects, the forearm link length $l_{\mathrm{ex}}$ of the exoskeleton must have been longer than their forearm - in fact, to such an extent that the linear compensation joint in the $U$ condition could not compensate for the difference (by moving further proximal). In order to adjust the difference in length, the only possibility for the system is to force an offset in $+x$ direction between the human and robot joints. This 'forced' offset also induces an offset in the exoskeleton's elbow rotary joint, which causes the measurement of $\beta$ to be offset from the true limb angle $\alpha$. Thus, the extension of the limb is not limited, but the sensing in the extended limb position is faulty. This can only happen for both kinematic configurations, if the compensation joint $\Delta_{8}$ does not offer sufficient stroke in proximal direction to compensate for the length-offset in the fully extended position of the limb. Only the identification of model parameters helped us to arrive at a suitable explanation of this effect. To improve the sensing accuracy as well as the available range of motion of the device, the joint design must be updated accordingly.

The statistical analysis of the force and torque measures has revealed a strong interaction between the levels of the kinematic setting and the subjects. This means that the subjects must have a different response to the $L$ and $U$ kinematic settings with respect to interface load creation. It can be seen in Figure 6 that subjects cause creation of interaction force (a) and torque (b) differently with respect to the kinematic setting. The question to solve is why the interaction force for some subjects is much lower in the $U$ condition, while for others it is not? This can be answered by considering that subject variation is apparent mainly as a variation between physiological parameters, which in turn causes variation in geometric attachment properties 
between the exoskeleton and each subject. Table 1 confirms that a main source of variability between the subjects can indeed be attributed to variation of their $\mathrm{ICR}_{\mathrm{h}}$ joint alignment to the exoskeleton $\mathrm{CR}_{\mathrm{e}}$. Even though we aimed at aligning the device well, still relatively large misalignments (from -2.2 to $+13.6 \mathrm{~cm}$ for $x$ and from 11.7 to $5.9 \mathrm{~cm}$ for $y$ ) occurred between the principal axes of the human and the robot. And offsets that exist during attachment are even amplified during movement. The offset-shifts during motion are indirectly caused by variation of anthropometry. Some subjects, for instance, had rather large upper-arm diameters, such that the exoskeleton upper-arm fixation could not be moved sufficiently towards the shoulder. The exoskeleton had to be attached at the level of the biceps, which typically caused positive $y$ offsets (in distal direction) between $\mathrm{ICR}_{\mathrm{h}}$ and $\mathrm{CR}_{\mathrm{e}}$. Some subjects (mainly the female ones S.7-S.14) had very thin upper arms such that the exoskeleton could be attached above the biceps, which was preferable for comfort (the exoskeleton not pressing onto the biceps). In that case, the contracting biceps appeared to move the exoskeleton's main axis proximally, showing as offsets in the $-y$ direction. On top of that, the link lengths of the subject's limbs varied significantly, which can affect offsets in the $x$ direction, as was outlined before. Clearly, it was not possible to control the variation of offsets better, without altering the experimental set-up to such extents as to lose equivalence to a real application. The different combinations of offsets cause different behaviours in the passive joints. This is the reason why different subjects experience different behaviours of interaction forces between the two kinematic settings. It can be seen in Table 1 that Subject 4, to which the exoskeleton fitted very well (very small offsets in both $x$ and $y$ directions), experienced the smallest interface loading in general (Figure 6a) for both kinematic conditions. The model identification therefore did not show good coefficient of determination. However, only a very small norm of the residuals exists. Interestingly, for all other subjects in Table 1 that have offsets in both $+x$ and $+y$ directions, significantly less force is created in the $U$ kinematic setting of the exoskeleton with respect to the $L$ setting (Figure 6(a)). For those subjects, the passive compensation joints in the EXARM worked better than for others. Subjects having offsets in $-x$ or $-y$ direction, or even in both directions, do not experience significantly less force in the $U$ condition. For them, the passive joints of the EXARM did not create sufficient reduction of forces. We aim at interpreting this result by consulting the pHRI model outputs presented in Figure 4 and the graphs presented in Figure 8.

We can see that if positive $x$ and $y$ offsets are present, force in the measured range of motion is mostly pointing in the positive direction (distal) near the motion end-range (where the peak forces are caused). The data presented in Figure 6(a) shows that this direction must be the preferred direction of motion of our linear compensation joint $\Delta_{8}$.
If forces with a positive sign can activate the linear compensation joint in the EXARM better, or more often, the force in the $U$ kinematics setting will be removed better as well. Forces from such combinations of offsets $(+x,+y)$ are larger in magnitude near the $90^{\circ}$ stroke-end (Figures 4(a) and 8(a)) than forces created by most other offsets. This means that in the $L$ kinematic setting, the measured force will be relatively high compared to the ones measured in the $U$ condition, which is what is shown by the significant results for the subjects in Figure 6(a). For the other offset combinations, differences between measured $L$ and $U$ forces were not sufficiently spread apart to show significance. For our exoskeleton device, we learn that the range of the passive joints needs to be updated. Then, the design will be more robust to variation between individuals.

To improve the design of the passive compensatory joints in general, we can state that sufficient margin of motion in both primary directions must be present at all limb positions.

The mitigation of torque loads in our design with passive joints is better. More subjects experience less torque in the $U$ condition (Figure 6(b)). Also, for the group (Figures 7(b) and 5(d)-(f)), the difference in interaction torques is more profound between the $L$ and $U$ settings. This indicates that the rotary passive joint does not have a preferred direction of motion and can thus compensate more interaction torques stemming from particular offsets of the individuals.

Another reason for the better force reduction in subjects with positive $x$ and $y$ offsets can also be the monotonically increasing, nearly linear, force characteristic over the workspace in this configuration. This characteristic enables an optimal combined functioning of the compensatory joints. All other subjects in Figures 8(b) and (c) experience a direction change of the force within the workspace. They have a less pronounced reduction of forces in the $U$ condition (Figure 6(a)). We hypothesise that a changing direction of force in the workspace causes disturbances that might be caused by additional dynamic effects between the human and the exoskeleton. The compensation joints can hit the stroke-ends more often during movement, which by themselves can create more peak forces. A linear force-position characteristic allows the passive compensation joints to work more smoothly and optimally. This hypothesis is supported by the fact that Subject 5 also, whose force characteristic is similarly linear, has a greatly reduced force in the $U$ condition, despite the fact that its offsets are in $+x$ and $-y$ directions.

The tracking performance during the experiments showed strong dependence on the capability of the subjects. This was expected. However, some subtle effects were revealed that were rather unexpected. At low attachment pressures from 10 to $30 \mathrm{mmHg}$, the subjects track the target signal better in the $U$ kinematic setting, whereas at higher pressures ranging from 40 to $60 \mathrm{mmHg}$, the subjects track the signal better in the $L$ condition. This raises the question 
that at which pressure the subjects did prefer to perform the tracking experiment?

When asked directly, the tested persons preferred to wear the exoskeleton at pressures between roughly 10 and $30 \mathrm{mmHg}$ overall. Even though a large range of pressures was tested, the preferred pressure of the group lies within a relatively narrow range. The mean subjective comfort ratings are highest for $20 \mathrm{mmHg}$ in the $U$ kinematic setting of the exoskeleton. This indicates a good optimum attachment pressure of about $20 \mathrm{mmHg}$. However, the subjects noticed only a comfort difference between the kinematic settings at $30 \mathrm{mmHg}$, where the comfort in the $U$ kinematic setting was rated higher. This allows us to summarise that low pressures along with the $U$ kinematic configuration of the exoskeleton feel more comfortable. This combination of settings also allows good signal-tracking performance. But why do the test persons prefer a low pressure for the task?

The weighting factors determined for the individual TLX rating scales show that the main workload of the task is associated with temporal demand, followed by effort. Effort rated by the human subjects increases with higher pressures. Also, the physical demand of the task increases with higher pressures. Even though the group preferred high pressures to reduce mental demand, the other effects are dominant, which is why, in summary, the physical demand and effort caused selection of a lower interface pressure at the expense of increased mental demand. However, if we consider our preferred selection of the $U$ kinematic setting for low pressures from the tracking results, we see that the mental demand ratings are also positively affected by the choice of kinematic configuration of the exoskeleton.

Pressure variation thus shows more effect on the subjective measures in our tracking experiment, whereas the kinematic variation shows more effect on the objective measures. Interestingly, the subjects did not, with the exception of mental load, clearly perceive the differences in kinematic setting. Since the pressure variation was the more dominant influence on subjective measures, it seems that underlying physical effects of lower interaction loads between kinematic settings of the exoskeleton were masked by the imposed pressure variation of the experiment.

However, we have clearly seen that interface loads are lower for the $U$ setting and have found a good way to optimise for ergonomics in our exoskeleton design by analysing measured forces of different individuals with a pHRI model.

In a motorised wearable robot, we believe, the differences of interface load will be even more profound and, depending on the task, also subjectively experienced. In particular for applications, where force-perception with high resolution and dynamic range is critical, e.g. in haptic devices, the inclusion of passive compensatory joints might improve the feel and mechanical transparency of the device. For such systems, a linear force characteristic would be ideal that enables an optimal functioning of the passive joints. Especially with regard to the level of typical force-feedback loads applied to the human joints, which are in the order of $1 / 20$ th of the maximum human joint strength, the elimination of interface force of up to $230 \mathrm{~N}$ and torques of up to about $1.5 \mathrm{Nm}$ seems utterly important. As an example, the exoskeleton presented in Tsagarakis and Caldwell (2003) transfers a torque of $6 \mathrm{Nm}$ to the elbow. The exoskeleton from Frisoli et al. (2005) can apply a force of $50 \mathrm{~N}$ cont. or $100 \mathrm{~N}$ peak to the tip of the hand. For robots that transfer large forces and torques, e.g. rehabilitation robots, the inclusion of compensation joints will mainly allow a larger range of movement and contribute to reducing safety-critical peak loads.

We can summarise that low attachment pressures, along with the $U$ kinematic setting of the exoskeleton, are optimal for comfort, signal-tracking performance, range of movement and low interaction loading. A low attachment pressure of $20 \mathrm{mmHg}$ is optimal for the subject group tested.

\section{Conclusion}

We thus conclude the following:

- Offsets between the axes of rotation of a human and a robot can easily be in the order of $\pm 10 \mathrm{~cm}$ in various directions, even if at the start of the movement, the two axes are well aligned.

- Such offsets create large interaction forces during movement between the attachment of a human operator limb and the robot limb that can peak at up to $230 \mathrm{~N}$ along the axis of the human limb. Large interaction torques around the attachment cuffs can be created that rise up to about $1.46 \mathrm{Nm}$.

- In conventionally designed exoskeletons, these interaction loads limit the voluntary range of motion near the stroke-ends.

- An attachment stiffness of about $300 \mathrm{~N} / \mathrm{m}$ is created at the human-robot interface.

- An exoskeleton that features passive compensation joints can lower such interaction forces by $70 \%$ and the torques by at least $60 \%$, and can allow about a $20 \%$ larger range of motion in the far stroke-end.

- For an exoskeleton device with passive joints to work optimally, its passive joints must feature sufficient stroke margin in both directions over the entire workspace.

- An exoskeleton with passive compensation joints can then be called 'ergonomic'.

- The optimum interface pressure between exoskeleton and human arm, from both subjective as well as objective points of view, is $20 \mathrm{mmHg}$ on both attachment cuffs.

- The best combination of subjective and objective performance for the task can be reached by an ergonomic exoskeleton that is attached with the optimum attachment pressure. 
In order to further improve the ergonomics of the ESA exoskeleton, we will update the implementation of the compensation joints, as learned from the analysis above. The joints need to be able to move over the entire workspace in both directions. The length of the upper arm and the forearm links of the exoskeleton will be re-designed in the next prototype to further improve robustness against physical parameter variations between subjects. The diameter of the upper-arm cuff will be increased to better fit to more muscular operators.

\section{Acknowledgement}

The authors would like to kindly thank all 14 volunteers who very enthusiastically participated in the experiment campaign.

\section{References}

Alami R, Albu-Schaeffer A, Bicchi A, Bischoff R, Chatila R, De Luca A, Santis AD, Giralt G, Guiochet J, Hirzinger G, et al. 2006. Safe and dependable physical human-robot interaction in anthropic domains: state of art and challenges. Presented at the International Conference on Intelligent Robots and Systems IROS, Beijing.

Bergamasco M, Allotta B, Bosio L, Ferretti L, Parrini G, Prisco M, Salsedo F, Sartini G. 1994. An arm exoskeleton system for teleoperation and virtual environments applications. Proceedings of the IEEE. Proceedings of International Conference on Robotics and Automation, 1994 12-14 May. Nice, France. Vol. 2, p. 1449-1454.

Carignan C, Tang J, Roderick S, Naylor M. 2007. A configurationspace approach to controlling a rehabilitation arm exoskeleton. Proceedings of 10th IEEE International Conference on Rehabilitation Robotics, p. 179-187.

Colombo G, Joerg M, Diez V. 2000. Driven gait orthosis to do locomotor training of paraplegic patients. Proceedings of 22nd Annual EMBS Conference, Chicago, IL. p. 23-28.

Colombo G, Joerg M, Schreier R, Dietz V. 2000. Treadmill training of paraplegic patients using a robotic orthosis. J Reliab Res Dev. 37(6):693-700.

Frisoli A, Rocchi F, Marcheschi S, Dettori A, Salsedo F, Bergamasco M. 2005. A new force-feedback arm exoskeleton for haptic interaction in virtual environments. Proceedings of $1 \mathrm{st}$ Joint EuroHaptics Conference and IEEE Symposium on Haptic Interfaces for Virtual Environment and Teleoperator Systems, Pisa, Italy. p. 195-201.

Hart SG, Staveland LE. 1988. Development of the NASA-TLX (Task Load Index), In: Hankock PA, Meshkati M, editors, Human mental workload. Elsevier Science Publishers BV: North Holland, p. 239-250.

Hidler JM, Wall AE. 2005. Alterations in muscle activation patterns during robotic-assisted walking. Clin Biomech. 20:184 193.

Hogan N. 1985. Impedance control: an approach to manipulation, Part I, II, II. J Dyn Syst Meas Control. 107:1-23.

Kazerooni H, Steger R. 2006. The Berkely lower extremity exoskeleton. J Dyn Syst Meas Control. 128(1):14-25.

Neckel ND, Nichols D, Hidler JM. 2007. Joint moments exhibited by chronik stroke subjects while walking with a prescribed physiological gait pattern. Proceedings of 10th IEEE International Conference on Rehabilitation Robotics, Noordwijk. p. 771-775.

Nef T, Mihelj M, Riener R. 2007. ARMin: a robot for patientcooperative arm therapy. J Med Biol Eng Comput. 45(9):887900.
Pratt G, Williamson M. 1995. Series elastic actuators. Procedings of IEEE/RSJ International Conference on Intelligent Robots and Systems, Pittsburg, USA. p. 388-406.

Raibert MH, Craig JJ. 1981. Hybrid position/force control of manipulators. ASME J Dyn Syst Meas Control. 102:275-282.

Riener R, Nef T, Colombo G. 2005. Robot-aided neurorehabilitation of the upper extremities. J Med Biol Eng Comput. 43:2-10.

Schiele A. 2008a. An explicit model to predict and interpret constraint force creation in p-HRI with exoskeletons. Proceedings of IEEE International Conference on Robotics and Automation (ICRA), p. 1324-1330.

Schiele A. 2008b. Case study: quantification of constraint displacements and interaction forces in non-ergonomic $\mathrm{pHR}$ interfaces. In: Pons JL, editor. Wearable robots: biomechatronic exoskeletons. John Wiley, New York, NY, USA. p. 149-154.

Schiele A. 2008c. Case study: the ergonomic EXARM exoskeleton. In: Pons J L, editor. Wearable robots: biomechatronic exoskeletons. John Wiley, New York, NY, USA. p. 248255.

Schiele A. 2008d. Fundamentals of ergonomic exoskeleton robots [Ph.D. thesis]. Delft, Netherlands: Delft University of Technology. Available at: www.library.tudelft.nl

Schiele A, De Bartolomei M, van der Helm F. 2006. Towards intuitive control of space robots: a ground development facility with exoskeleton. Proceedings of International Conference on Intelligent Robots and Systems, IEEE/RSJ, Beijing, China. p. 1396-1401.

Schiele A, van der Helm FCT. 2006. Kinematic design to improve ergonomics in human machine interaction. IEEE Trans Neural Syst Rehabil Eng. 14(4):456-469.

Tsagarakis NG, Caldwell DG. 2003. Development and control of a 'soft-actuated' exoskeleton for use in physiotherapy and training. Autonom Robots 15:21-33.

Zinn M, Roth B, Khatib O, Salisbury JK. 2004. New actuation approach for human-friendly robot design. Int J Robot Res. 23(4-5):379-398.

Zoss A, Kazerooni H. 2006. Design of electrically actuated lower extremity exoskeleton. Adv Robot. 20(9):967-988.

\section{Appendix}

The pHRI model derives the total displacement $d_{\text {tot }}$ along the movable link in dependence of $\alpha$ and $\beta$ according to

$$
\begin{aligned}
& d_{\mathrm{tot}}\left(\alpha, x, y, l_{\mathrm{ex}}, z_{\mathrm{ex}}\right)=l_{\mathrm{ex}} \cdot \sqrt{1-\frac{x^{2}}{l_{\mathrm{ex}}^{2}}}+y \\
& -\frac{\sqrt{x^{2}+y^{2}} \cdot z_{\mathrm{ex}} \cdot \cos [\alpha-\arctan (y / x)]}{l_{\mathrm{ex}} \cdot \sqrt{1-\frac{\left(x^{2}+y^{2}\right)}{l_{\mathrm{ex}}^{2}} \cdot \cos [\alpha-\arctan (y / x)]^{2}}} \\
& -\sqrt{\psi^{2}+\psi^{2} \cdot \tan [\alpha]^{2}},
\end{aligned}
$$

with

$$
\begin{aligned}
\psi & =y-l_{\mathrm{ex}} \cdot \sin \\
& \times\left(\alpha+\arcsin \left(\frac{\sqrt{x^{2}+y^{2}} \cdot \cos [\alpha-\arctan (y / x)]}{l_{\mathrm{ex}}}\right)\right. \\
& -\arctan (y / x)-\arctan (x / y)) .
\end{aligned}
$$




$$
\begin{aligned}
d_{\mathrm{tot}}\left(\beta, x, y, l_{\mathrm{ex}}, z_{\mathrm{ex}}\right)= & l_{\mathrm{ex}} \cdot \sqrt{1-\frac{x^{2}}{l_{\mathrm{ex}}^{2}}}+y-\sqrt{\left(y+l_{\mathrm{ex}} \cdot \cos (\beta)\right)^{2} \cdot \operatorname{Csc}\left(\beta+\arccos \left(\frac{\sqrt{x^{2}+y^{2}} \cdot \sin [\beta+\arctan (x / y)]}{\sqrt{\Upsilon}}\right)\right)^{2}} \\
& -\frac{\sqrt{x^{2}+y^{2}} \cdot z_{\mathrm{ex}} \cdot \sin [\beta+\arctan (x / y)]}{\sqrt{\Upsilon} \cdot \sqrt{1-\frac{\left(x^{2}+y^{2}\right) \cdot \sin [\beta+\arctan (x / y)]^{2}}{\Upsilon}}}
\end{aligned}
$$

with

$\Upsilon=l_{\text {ex }}^{2}+x^{2}+y^{2}+2 \cdot l_{\text {ex }} \cdot \sqrt{x^{2}+y^{2}} \cdot \cos [\beta+\arctan (x / y)]$. 

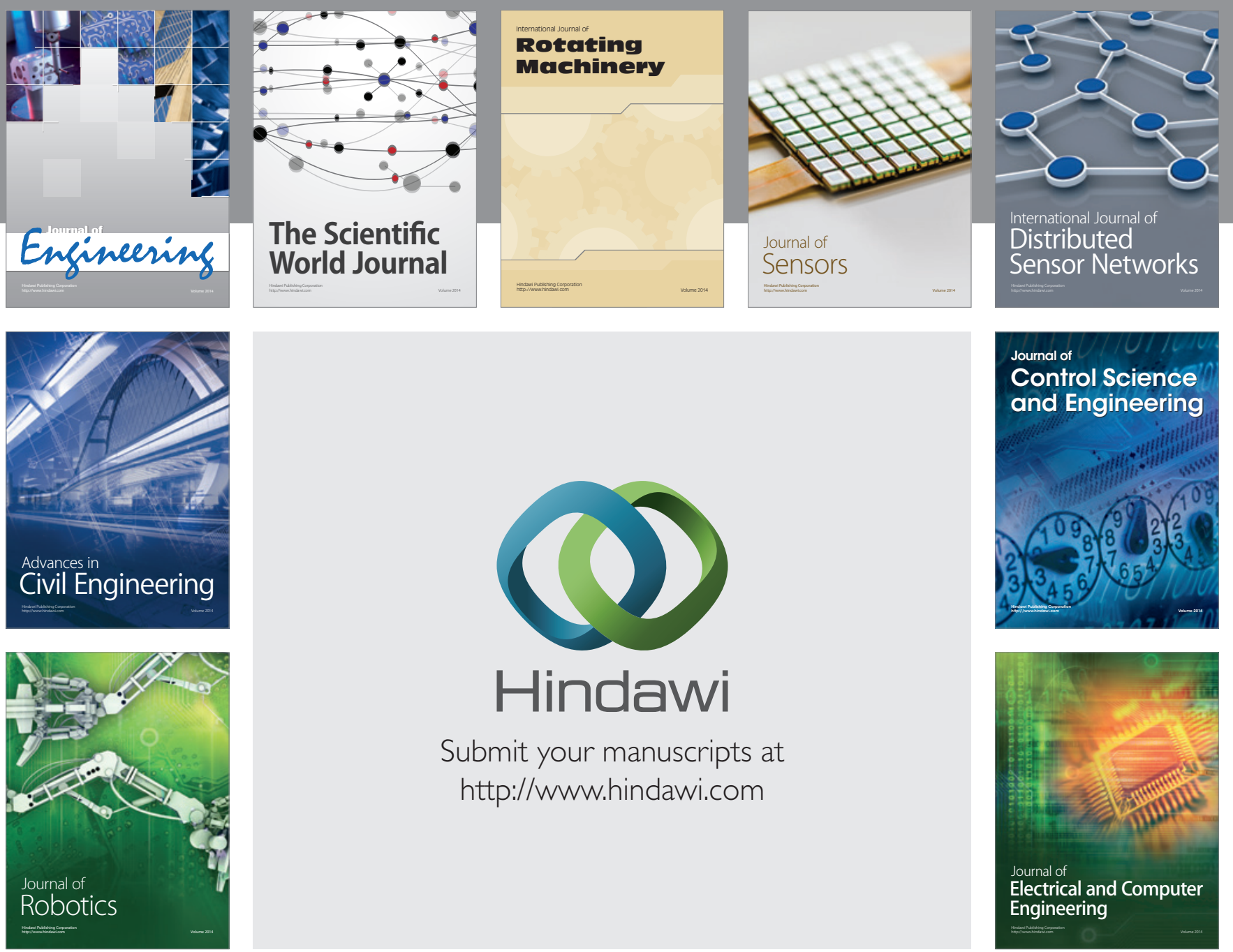

Submit your manuscripts at

http://www.hindawi.com
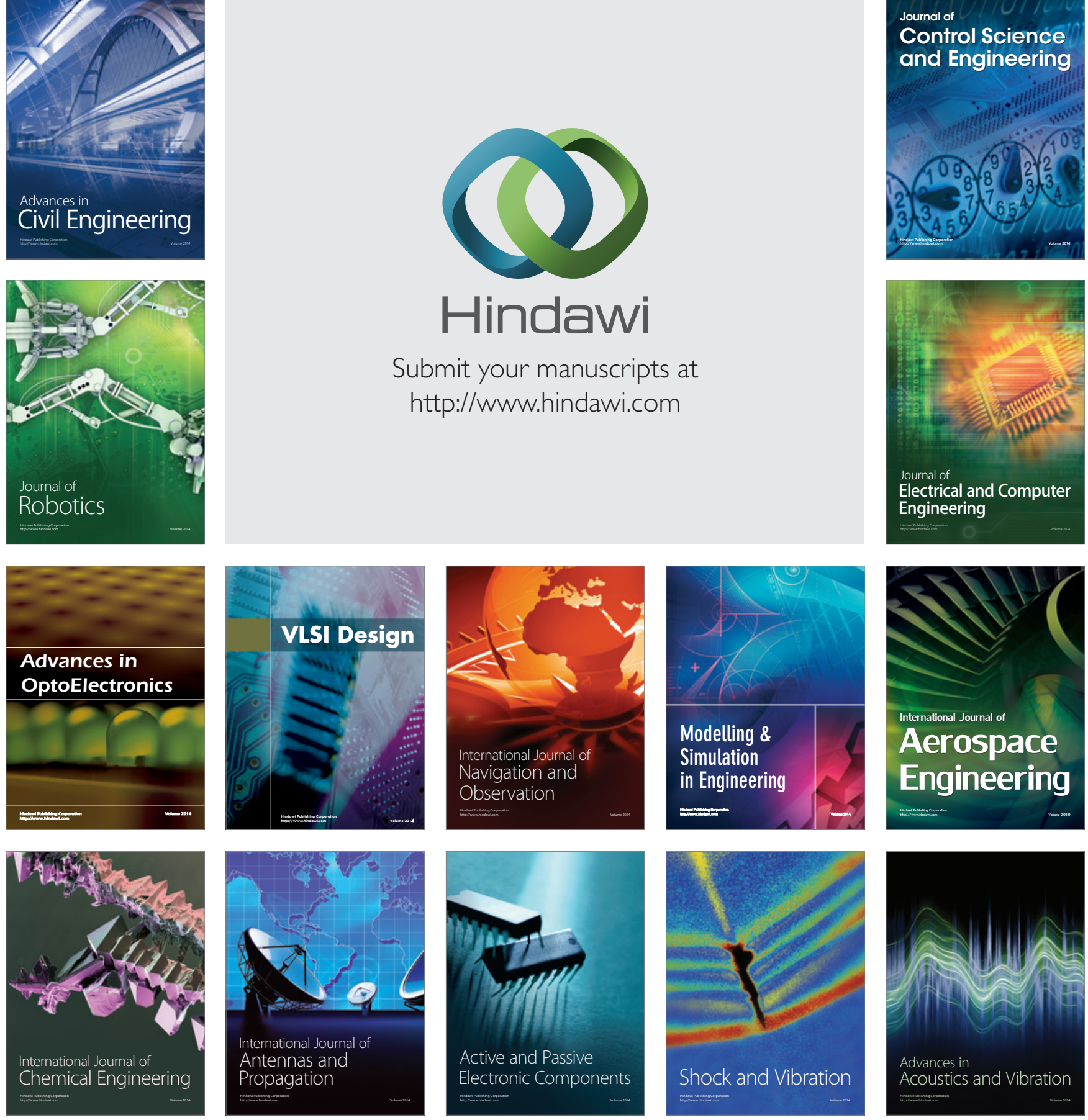